\title{
Increase in suicide following an initial decline during the COVID-19 pandemic in Japan
}

\author{
Takanao Tanaka $\oplus^{1}$ and Shohei Okamoto $\circledast^{2 凶}$
}

\begin{abstract}
There is increasing concern that the coronavirus disease 2019 (COVID-19) pandemic could harm psychological health and exacerbate suicide risk. Here, based on month-level records of suicides covering the entire Japanese population in 1,848 administrative units, we assessed whether suicide mortality changed during the pandemic. Using difference-in-difference estimation, we found that monthly suicide rates declined by $14 \%$ during the first 5 months of the pandemic (February to June 2020). This could be due to a number of complex reasons, including the government's generous subsidies, reduced working hours and school closure. By contrast, monthly suicide rates increased by $16 \%$ during the second wave (July to October 2020 ), with a larger increase among females (37\%) and children and adolescents (49\%). Although adverse impacts of the COVID-19 pandemic may remain in the long term, its modifiers (such as government subsidies) may not be sustained. Thus, effective suicide prevention-particularly among vulnerable populations-should be an important public health consideration.
\end{abstract}

$\mathrm{T}$ he COVID-19 pandemic has affected every aspect of life. As the virus has spread globally ${ }^{1}$, anxious individuals have voluntarily engaged in physical distancing and reduced their economic activities to prevent infection. To contain the virus, governments have implemented large-scale costly interventions in an unprecedented manner: citizens and communities have been requested to limit social contacts, avoid social gatherings, close schools and stop unnecessary business activities. To date, most scientific and clinical attention has been given to identifying the disease's direct physical risk $^{2,3}$ and its prevention ${ }^{4-6}$. However, the end of this pandemic remains a distant prospect. This raises an emerging concern in a different public health arena: the pandemic could adversely affect people's mental health ${ }^{7,8}$ and-in a more pressing scenario-deaths from suicide could increase ${ }^{9,10}$.

While suicide is rarely caused by a single factor, and reasons for changes to suicide prevalence are extremely complex, previous studies suggest that the pandemic could affect the suicide rate in various ways. Together with fear, uneasiness and anxiety caused by the threat of the disease, social distancing can lead to impaired social and family relationships; increased loneliness, boredom and inactivity; and restricted access to healthcare services, potentially inducing mental illness and increased suicidal behaviours ${ }^{10,11}$. Financial insecurity and loss of employment are well-known risk factors for suicide $^{12,13}$. Thus, the pandemic-driven economic recession could increase death from suicide ${ }^{14}$. Unsurprisingly, existing studies suggest that past epidemics such as the Spanish Flu and severe acute respiratory syndrome led to increased suicide rates ${ }^{15,16}$. At the same time, the current pandemic might have reduced some of the stress from workplaces and social interactions (such as commuting or bullying in school) and government financial support could have partially alleviated the pandemic's adverse impacts. However, given the unprecedented magnitude, ubiquity and complexity of the ongoing public health crisis, adequate preventive measures to reduce the risk of suicide will be required. To formulate effective policy responses, policymakers, healthcare professionals and researchers need a credible assessment of suicide prevalence during the pandemic.
However, reliable empirical evidence regarding the link between the COVID-19 pandemic and suicide mortality remains scarce. An inclusive assessment requires harmonised data that cover representative and sufficiently large samples but are collected at a disaggregated level ${ }^{17,18}$. Such data should also include information from both the pre-COVID-19 period (to serve as the baseline) and the COVID-19 period. However, existing studies use readily available and convenient data that could easily generate biased insights; many studies rely on some measures of suicidality rather than suicide mortality ${ }^{19-24}$ and most of them compare suicidal behaviours using snapshot data during the pandemic without pre-pandemic baseline samples ${ }^{19,20,22,23}$. Even when studies use real suicide mortality, some rely on data that cover non-representative subsamples ${ }^{25-27}$, while others compare the whole suicide trend before and during the pandemic, which might capture common time trend, seasonality or temporal time shocks across individuals or locations ${ }^{25-31}$ (we discuss why these time-series analyses and before-after comparisons can be problematic in the Methods).

In this study, we provide large-scale evidence linking the COVID19 outbreak to suicide fatalities using a city-by-month-level dataset covering the entire Japanese population of more than 120 million people (Fig. 1; details of the data are provided in Supplementary Note 1 and Supplementary Table 1). Since the confirmation of the first case of COVID-19 in Japan, the nation has been hit by two large-scale outbreaks. In response, national and local governments have implemented various preventive interventions, such as nationwide school closure and state of emergency (SOE), and local business restrictions. Although the government concurrently provided generous financial support for citizens and enterprises, accounting for as much as $10 \%$ of Japan's annual GDP ${ }^{32}$, people's lives were still profoundly affected; for instance, geographic mobility fell below the pre-pandemic level and the unemployment rate increased for nine consecutive months (Supplementary Fig. 1 and Supplementary Note 2). Since the Japanese suicide rate is the seventh highest among high-income countries ${ }^{33}$ and suicide has been among the top ten causes of death in Japan for the last two

'Division of Social Science, Hong Kong University of Science and Technology, Clear Water Bay, Hong Kong. ${ }^{2}$ Research Team for Social Participation and Community Health, Tokyo Metropolitan Institute of Gerontology, Tokyo, Japan. $\varpi_{e}$-mail: sokamoto@tmig.or.jp 

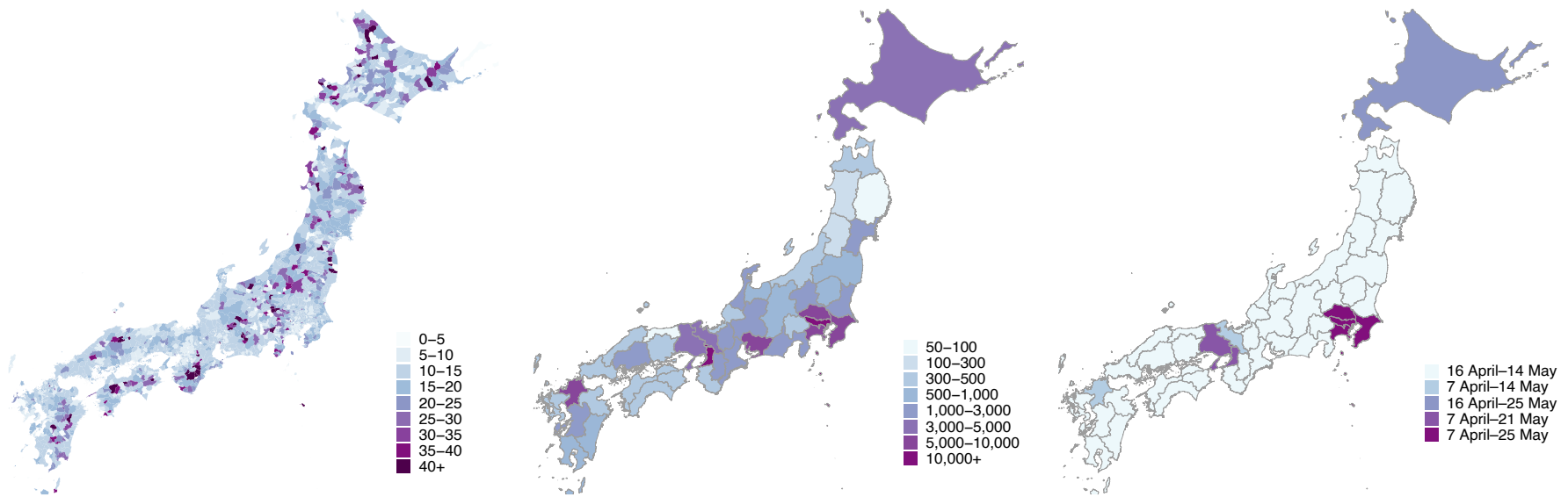

Fig. 1 | Distribution of suicide rate, COVID-19 and health interventions in Japan. a, Mean monthly suicide rate (per million) in each city during the period of our study. b, Number of confirmed COVID-19 cases on October 31 in each prefecture. c, Dates of the SOE implemented and lifted during the COVID-19 outbreak. Maps were created by the authors using the shape file (details in Supplementary Note 1).

decades $^{34}$, there have been growing concerns that the COVID-19 crisis may increase suicide deaths.

Our data have some notable advantages for assessing whether suicide mortality changed during the pandemic and the subsequent health interventions. First, our data cover both pre-pandemic and pandemic-era samples from November 2016 to October 2020, so we can investigate the relative change in suicide rate compared with the pre-pandemic baseline (we describe the suicide trend in Extended Data Figs. 1 and 2). In particular, to construct a reliable control condition (without pandemic), we use a difference-in-differences (DID) estimation. We assess whether suicide rates during the pandemic varied compared with the corresponding seasons in previous years, after controlling for the variation in the overall suicide level across years. Moreover, our data are collected from 1,848 administrative units, which enables us to control for various confounding factors. Specifically, our regression includes two sets of high-dimensional fixed effects: a city-by-month fixed effects control for month-specific shocks in each city (for example, seasonality in suicide rate, monthly local events or climatic conditions), and a city-by-year fixed effects control for a year-specific shock in each city (for example, macroeconomic trend, industrial or population structural changes or suicide trends) (we describe details of this regression in the Methods).

The effects of the pandemic might not be evenly distributed across populations. To identify the vulnerable populations, we further analyse heterogeneous impacts across gender and age groups. Historically, male adults have faced the highest suicide risk in Japan. During the pre-pandemic period in our dataset, the male suicide rate is about 2.3 times as high as that of females (per-month suicide rate is 18.1 per million for males and 7.8 per million for females from November 2016 to January 2020 (Supplementary Table 1)), and is even higher among male adults (21.8 per million among males aged 20-69 years). Because previous literature suggests that financial and economic distress could trigger suicide fatalities particularly among males $^{35}$, and the COVID-19 pandemic brought about non-negligible disruptions in the labour market, we might expect its adverse impact to be greater among such populations (Supplementary Note 3). Alternatively, older adults face an increased risk of infection and death from COVID-192,3, which could amplify their fear and distress about virus transmission. Additionally, existing studies suggest that unlike normal economic recessions, this pandemic has gendered impacts: social distancing disproportionately affects female-dominant employment ${ }^{36}$, and stay-at-home orders increase household tasks and domestic violence, which could disproportionately impair wellbeing among females ${ }^{37}$. These suggest that the effects of the COVID-19 pandemic could be intensified among these previously lower-risk populations.

Two further analyses complement our empirical analyses. First, we investigate whether the impacts vary across job status, which can indicate channels through which the pandemic and health interventions can affect suicide mortality. For instance, we might expect government subsidies to mitigate the adverse impacts differently across individuals (such as employed, retired and unemployed) and company owners (self-employed). Further, household dynamics (such as increase in housework or domestic violence) might disproportionately affect housewives' psychological health, and unusual school calendars (nationwide school closure and the resulting reductions in outdoor activities and social connections) might affect students' mental wellbeing. Finally, we assess whether the effects of the pandemic varied across different types of cities using the dimensions of original suicide risks, pandemic-associated risks (COVID-19 prevalence, health interventions and economic downturns), and base socio-economic status (income and urban populations). For example, we investigate whether the suicide trend varied between cities with high and low per capita income.

\section{Results}

Main results. The suicide rate declined substantially during the first wave of the COVID-19 pandemic (February to June 2020), but increased rapidly during the second outbreak (July to October 2020) (Fig. 2a). The DID estimates during the first outbreak-adjusting for permanent unobserved city-by-year and city-by-month determinants of the suicide rate-show that the overall suicide rate declined by $14 \%$ (incidence rate ratios $($ IRR $)=0.86,95 \%$ confidence interval (CI) 0.82 to 0.90 ) compared with the same season in previous years. By contrast, the suicide rate increased by $16 \%$ (IRR $=1.16,95 \% \mathrm{CI}$ 1.11 to 1.21 ) during the second outbreak (Fig. 2a). An increasing suicide trend was apparent during the second wave, and the suicide rate increased by $38 \%$ in October 2020 (IRR $=1.38,95 \%$ CI 1.27 to 1.49; full results are presented in Supplementary Tables 2 and 3).

Using the event-study approach, from estimated coefficients, we confirm that the assumption for parallel trends is not violated during the pre-treatment period, as average suicide rates in 2016-2019 and 2020 are not different (Fig. $2 ; k \leq-1$ ) (Supplementary Table 4). 

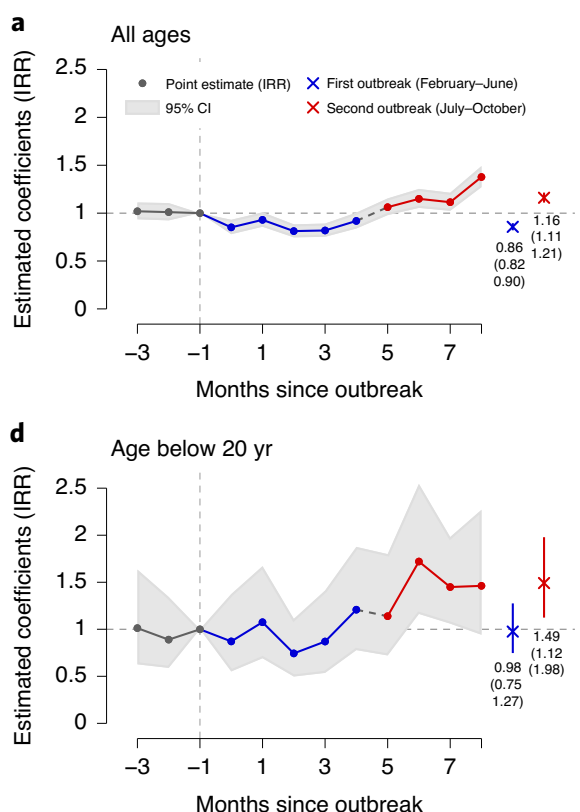
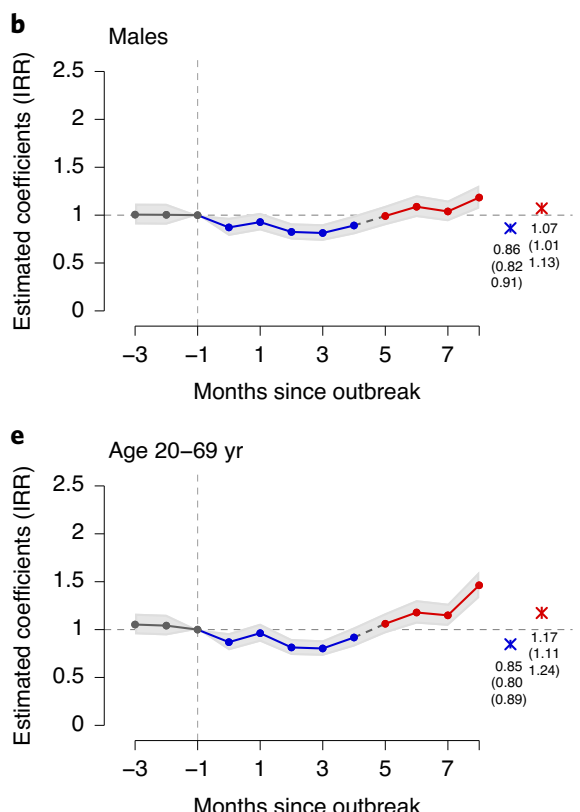
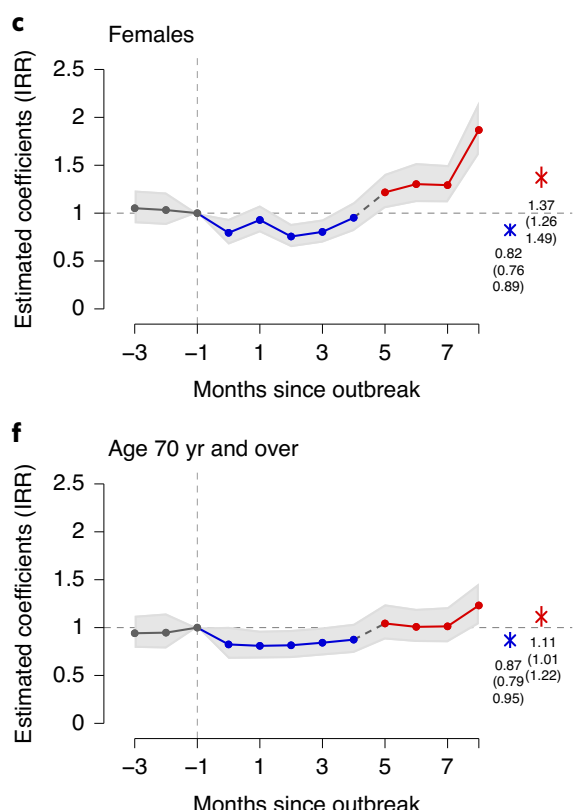

Fig. 2 | The effects of COVID-19 pandemic on suicide across gender and age groups using DID and event-study approaches. a, Results of the DID and the event study using all pooled samples. b,c, Results of comparing suicide rates among males (b) and females (c). d-f, Results for different age groups: below 20 years (d), 20-69 years (e) and $\geq 70$ years (f). Lines represent point estimates before the pandemic (grey), during the first outbreak of COVID-19 (blue) and during the second outbreak (red), with shaded areas showing the $95 \% \mathrm{Cl}$. Blue and red crosses show the DID estimates (and $95 \% \mathrm{CI}$ ) during the first and second outbreaks, respectively. Full results are presented in Supplementary Tables 2-4. All regressions include city-by-year fixed effects and city-by-month fixed effects and are weighted by the population. Standard errors are clustered at the city level. N=61,209 (a), 54,583 (b), 35,712 (c), 1,896 (d), 53,164 (e) and 34,703 (f). The separated observations are excluded (see Methods), and suicide data for children and adolescents are aggregated at prefectural level.

These results are robust to the inclusion of the time-varying weather variables and prefecture-specific quadratic time trends (Supplementary Fig. 2a). Moreover, the adoption of the OLS model, instead of the Poisson model, does not affect our main findings (Supplementary Fig. 2b).

To confirm that the results are not driven by either the common time trends or common year-specific shocks across cities or regions, we conducted a placebo test. Extended Data Fig. 3a shows that when using the placebo samples, the estimated coefficients are close to zero, and our real estimate for the first outbreak is much smaller than the lower bound of the coefficients' 95\% CI (IRR 0.96 to 1.04), while the estimate for the second outbreak is much larger than the upper bound of the coefficients' 95\% CI (IRR 0.96 to 1.04). This analysis suggests that our results are not driven by a spurious correlation. These patterns hold when we conduct the event-study regression analogously using the placebo samples, validating the strong relationship between the pandemic and the suicide rate (Extended Data Fig. 3b).

On average, 1,596 individuals died by suicide in each month during our study period (November 2016 to October 2020). Our rough calculation finds that there were 1,074 averted suicide deaths during the initial outbreak (95\% CI 830 to 1,488 ) from February to June 2020 but there were 970 additional suicide deaths during the subsequent outbreak (95\% CI 630 to 1,170) from July to October 2020 (Extended Data Fig. 4). In the corresponding period, the number of direct deaths from COVID-19 was 1,765 (as of October 31), suggesting that the suicide effect of the pandemic is sizeable by comparison.

Heterogeneity across gender and age groups. We investigated the heterogeneous impacts of the pandemic across gender, age groups and periods and found some noteworthy variations in the magnitude of the pandemic effects (full results in Supplementary Table 5).
First, during the nationwide SOE, there was a marked reduction in suicide among adults (individuals aged 20-69 years) (Fig. 3a). In the middle of the first wave, to slow down viral transmission, the national government declared an SOE, during which individuals were requested to stop unnecessary business, reduce social contacts and stay at home if possible. During this period, suicide deaths among adults declined by about $21-27 \%$ (Fig. 3a; male adults, $\mathrm{IRR}=0.79,95 \% \mathrm{CI} 0.73$ to 0.85 ; female adults, $\mathrm{IRR}=0.73,95 \% \mathrm{CI}$ 0.65 to 0.83 ); this was about twice the reduction in suicide deaths during other periods in the first wave. By contrast, the reduction in the suicide rate among individuals aged 70 years and over was not substantially larger during the SOE (Fig. 3a,b).

Second, the increase in suicide fatalities in the second wave was driven primarily by females, children and adolescents (individuals aged below 20years) (Figs. 2 and 3). Suicide mortality increased by $37 \%$ among females (Fig. 2 c; IRR $=1.37$, $95 \%$ CI 1.26 to 1.49 ), about 5 times more than the effect in males (Fig. 2b; IRR $=1.07,95 \%$ CI 1.00 to 1.14 ). This increase was most pronounced in October 2020, when the suicide rate among females increased by $82 \%$ (Supplementary Table 3; IRR $=1.82$, 95\% CI 1.62-2.04). The suicide rate among children and adolescents also increased in the second wave, which mostly corresponds to the period after the end of the nationwide school closure (Fig. 2d; IRR $=1.49,95 \%$ CI 1.12 to 1.98 ).

Heterogeneity across employment status. The suicide rate decreased among employed, retired and unemployed individuals in the first wave of the epidemic, but mostly increased in the second wave (Fig. $4 \mathrm{a}-\mathrm{c}$ ). These patterns are identical to the population as a whole. By contrast, we observed differences in suicide trends among specific sub-populations.

Thesuiciderateamongtheself-employed didnotdeclinein eitherthe first or second wave (Fig. 4d). By contrast, suicides among housewives 


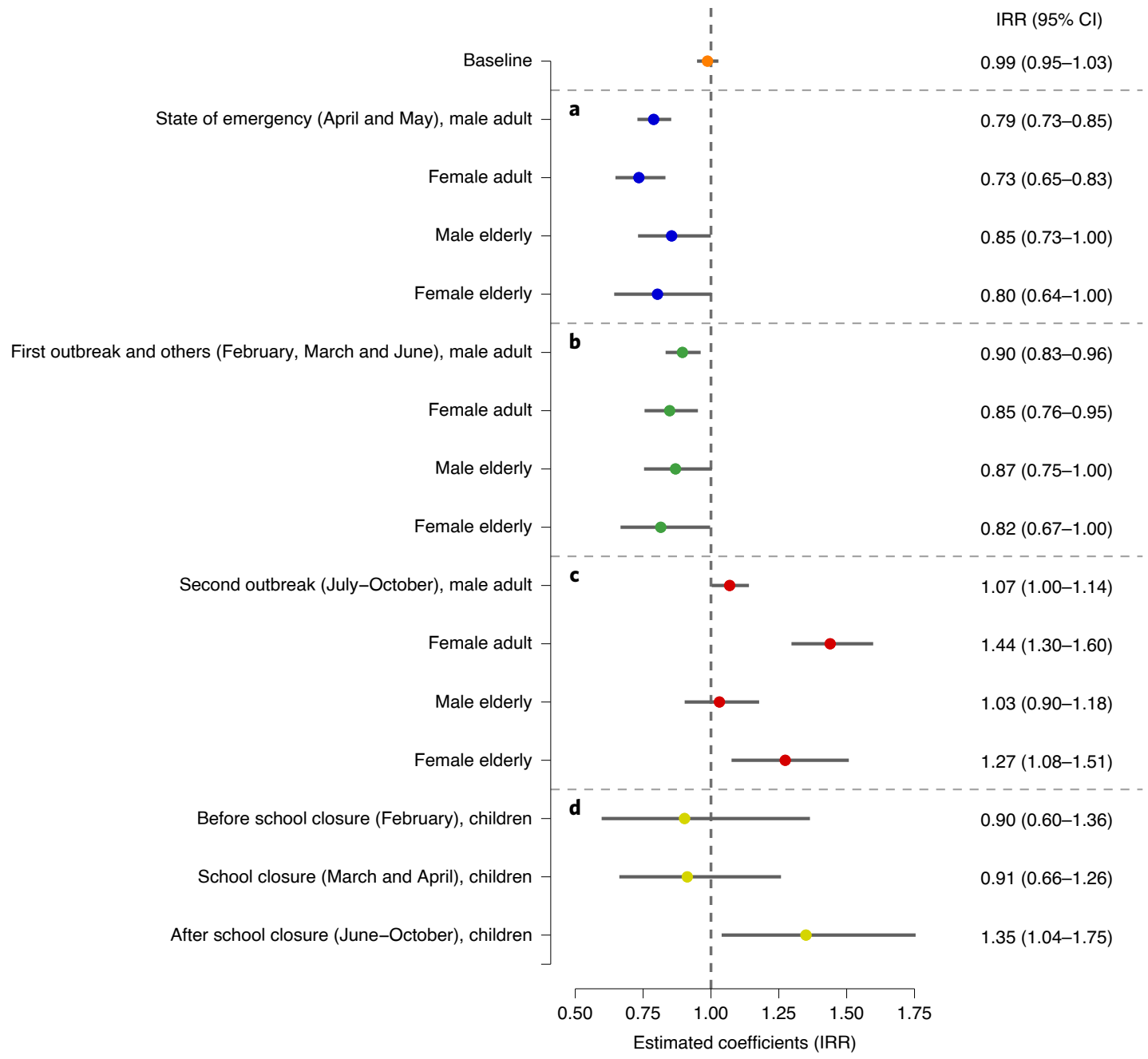

Fig. 3 | Heterogeneous effects of the COVID-19 pandemic with age groups and gender, before and after the state of emergency and school closure. a-d, Here we assign April and May as the period of the SOE, February-June as the first outbreak period and July-October as the second outbreak period. The baseline is the result for the pooled data. The circle and line denote the DID result and its $95 \% \mathrm{Cl}$. $\mathbf{a}$, Heterogeneous effects among age groups and gender during the SOE. b,c, Heterogeneity across age and gender groups during the first outbreak (excluding the SOE) (b) and second outbreak (c). d, Effects on students during school closure (March and April) and other periods. Full results are presented in Supplementary Table 5. All regressions include city-by-year fixed effects and city-by-month fixed effects and are weighted by the population; standard errors are clustered at the city level. $N=61,209$ (all), 47,317 (male adults), 26,319 (female adults), 24,478 (male elderly), 16,531 (female elderly) and 1,896 (children and adolescents). The separated observations are excluded (Methods), and suicide data of children and adolescents are aggregated at prefectural level.

(defined as adult women who are married and not employed in wage labour) increased during all pandemic periods (Fig. 4e). In particular, from July to October 2020 , the rate increased by $132 \%(\mathrm{IRR}=2.32$, $95 \%$ CI 1.65 to 3.26 ). Suicide deaths among students fell by about $49 \%$ during the school closure period (Fig. 4f; IRR $=0.51,95 \% \mathrm{CI}$ 0.34 to 0.77 ) (full results in Supplementary Table 6). The results for students were slightly different than those for children and adolescents. We describe these data in Supplementary Note 1.

Heterogeneity across cities. We investigate whether the effect of the pandemic varied across different types of cities (Fig. 5; full results in Supplementary Table 7). First, we compared cities with higher suicide risks (if the suicide rate before the pandemic was high, the city is classified as a high-suicide-risk city) with cities with lower suicide risks (Fig. 5a). We might expect suicide effects during the pandemic to be intensified in the high-risk cities. However, the results show the opposite effect; only cities that previously had low suicide rates showed an increase during the pandemic.
We also investigated heterogeneity with respect to disease-associated risks. We used COVID-19 prevalence (measured by total confirmed cases per million population in October 2020) and found that the effects on suicide deaths did not vary across cities (Fig. 5b). We also investigated variations in intensity of the health intervention (Extended Data Fig. 5a; measured by the changes in Google Community Mobility Reports (https://www.google. $\mathrm{com} /$ covid19/mobility/) at workplaces from January 2020 to after February 2020) and economic shocks (Extended Data Fig. 5b; measured by the changes in unemployment from October-December 2019 to April-September 2020), and did not find notable disparities in the impact on suicide deaths. Note that because these data are available at prefectural level, but not at city level, these results should be interpreted with caution.

Finally, the effects were not heterogeneous in terms of the base socio-economic status, such as income per capita (Fig. 5c; measured in 2018) or urban population (Extended Data Fig. 5c; measured in 2018). 

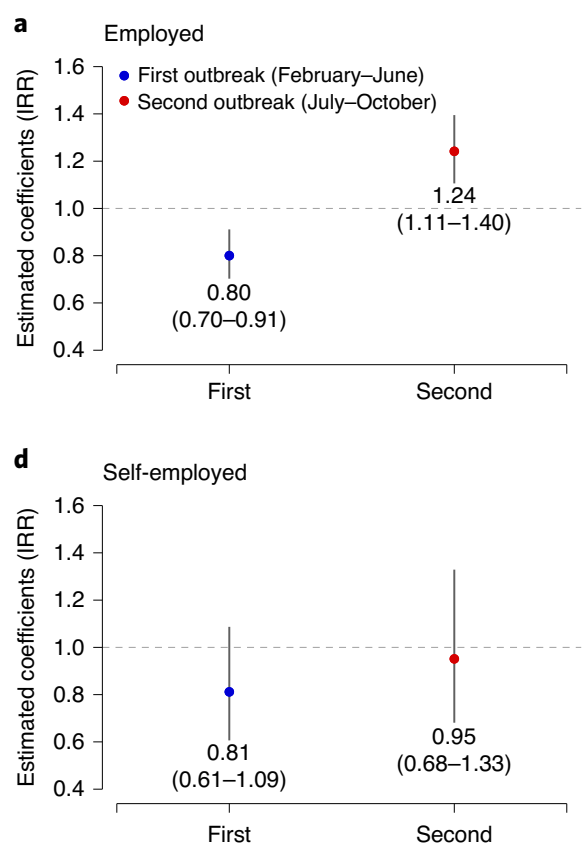
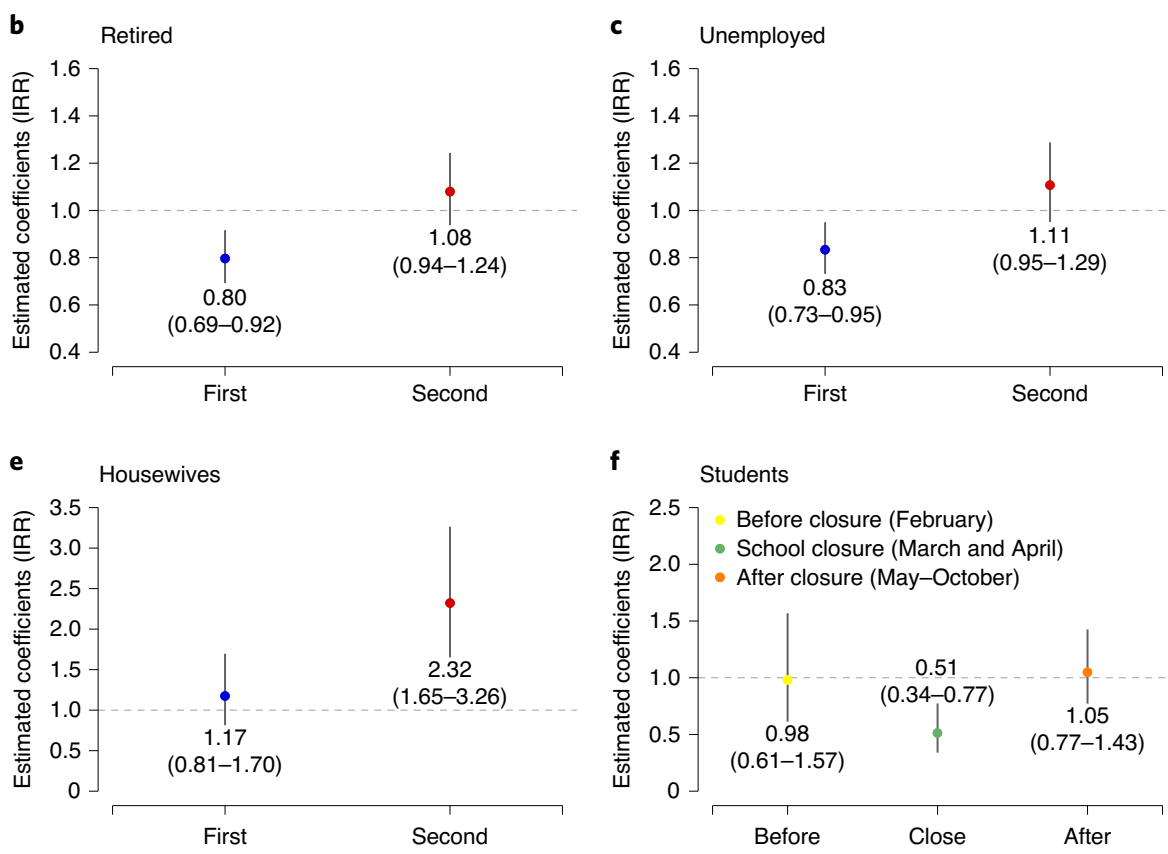

Fig. 4 | Heterogeneous effects of the COVID-19 pandemic according to job status. a-f, Effects of the pandemic on suicide rate across individuals with different employment statuses during the first and second COVID-19 outbreaks and during school closure for students. In a-e, blue and red circles denote the DID estimates during the first and second outbreaks, respectively, and error bars show $95 \% \mathrm{Cl}$. a-f, Suicide rate among employed (a), retired (b), unemployed (c), self-employed (d), housewives (e), and students (f). Full results are presented in Supplementary Table 6. All regressions include city-by-year fixed effects and city-by-month fixed effects and are weighted by population, and standard errors are clustered at the city level. $N=10,723$ (a), 9,146 (b), 9,246 (c), 4,124 (d), 3,854 (e) and 3,220 (f). The separated observations are excluded (Methods). Data used for this analysis are described in Supplementary Note 1.

\section{Discussion}

Using high-frequency data covering entire Japanese population, we investigated whether suicide mortality changed during the pandemic. In Methods, we show how the use of time-series analysis ${ }^{25-31}$ and simple before-after comparison ${ }^{26-30}$ could generate biased estimates. Instead, we used a DID model with high-dimensional fixed effects that allows us to control for a number of potential confounders. Consistent with emerging concerns, we find that suicide deaths increased from July to October 2020 (IRR $=1.16,95 \%$ CI 1.11 to 1.21 ), although the effects appeared several months after the pandemic started. Notably, an increasing suicide trend was apparent during this period.

The COVID-19 pandemic has affected every aspect of life in Japan. The unemployment rate has increased for nine consecutive months since the epidemic was recognised (Supplementary Fig. 1c). Social interactions and mobility also have been substantially restricted by individual choice or government intervention (see mobility index in Supplementary Fig. 1d). Fear and anxiety regarding infection are persistent. Given these circumstances, although the reasons behind suicide are extremely complex, there are a number of factors that could explain the rapid increase in the suicide rate during the second COVID-19 outbreak.

We find that the effects of the pandemic are not evenly distributed across populations and that there are differences with the historical suicide. First, the previous suicide rate among males was 2.3 times higher than among females (from November 2016 to January 2020; Supplementary Table 1), and the increase in suicide among males after previous financial crises was larger than that among females ${ }^{38}$. By contrast, during the second wave of the COVID-19 pandemic the increase in the suicide rate among females (IRR $=1.37,95 \% \mathrm{CI}$ 1.26 to 1.49 ) was about five times greater than that among males $(\mathrm{IRR}=1.07,95 \% \mathrm{CI} 1.01$ to 1.13$)$, with a larger increase among housewives (IRR $=2.32,95 \%$ CI 1.65 to 3.26 ). The suicide level for males remained higher than for females, but disparities decreased. These results are consistent with recent studies that find that this crisis has had a larger effect on female-dominant industries ${ }^{36}$, and that stay-at-home orders magnify the working mother's burden ${ }^{39}$. Similar patterns were observed in the Japanese labour market: the decrease in female employment was more pronounced than the decrease in male employment (Fig. 6a), and there was a larger effect on non-regular workers (56\% of females and $22 \%$ of males are non-regular workers) (Fig. 6b). In addition, domestic violence, which harms mostly women (more than $95 \%$ of all cases), increased (Fig. 6c and Supplementary Note 1). All of these factors may have contributed to harming women's psychological health.

The suicide rate among children and adolescents also increased in the second wave of the pandemic (IRR $=1.49,95 \%$ CI 1.12 to 1.98). This may be because the pandemic also excessively affects younger workers, who are more likely to be low-skilled and employed on less secure work contracts. Indeed, the decline in the employment rate during the disease outbreak was greater among this age group. Furthermore, the timing of the second outbreak corresponded to the period when schools (elementary school to high school) were reopened after the nationwide school closure. Previous studies have reported that schooling could be a risk factor for violence ${ }^{40}$ and suicide ${ }^{41}$ among students. After a period of a few months without school during the pandemic, the stress from returning to school could have been exacerbated. These factors may have amplified children's and adolescents' psychological depression.

Immediately after the COVID-19 outbreak (February to June 2020), however, we find a notable reduction in suicide rates (IRR $=0.86,95 \%$ CI 0.82 to 0.90 ). The finding may have been unexpected, but it is consistent with emerging studies and statistics showing that suicide deaths decreased in Norway ${ }^{30}$, the $\mathrm{UK}^{42}$, Germany ${ }^{27}$, 


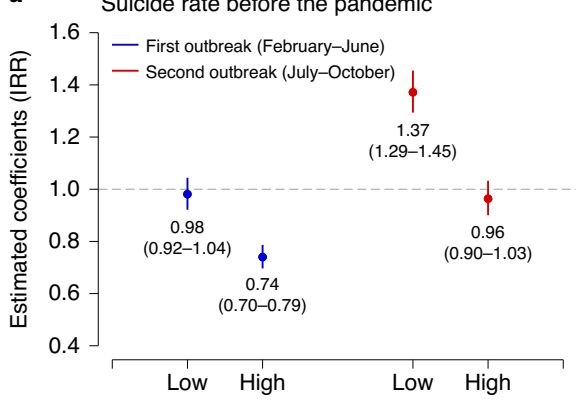

b

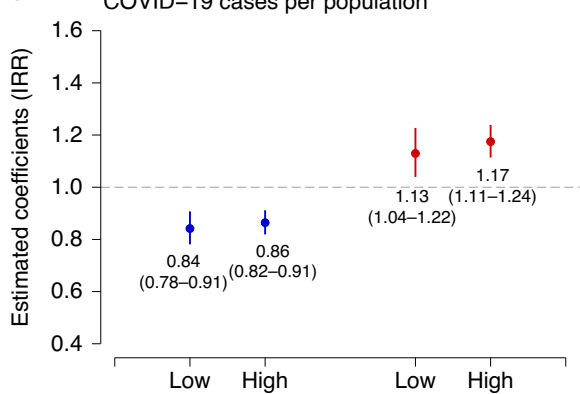

C Base income per capita

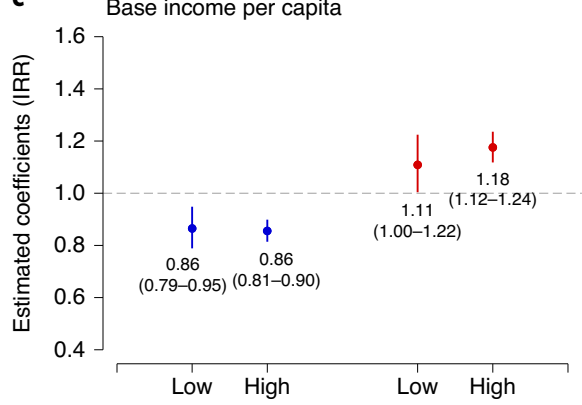

Fig. 5 | Heterogeneous effects of the COVID-19 pandemic across geography. a-c, Effects of the pandemic on suicide rate during the first and second outbreaks across locations with different original suicide risk before the pandemic (a), different prefectural level confirmed COVID-19 cases (b), and different base income per capita (c). Blue and red circles denote the DID estimates during the first and second outbreaks, respectively, and error bars show $95 \% \mathrm{Cl}$. If the variable in a city or a prefecture is lower than the median, the city or the prefecture is assigned to the low group; otherwise, it is in the high group. Full results are presented in Supplementary Table 7. All regressions include city-by-year fixed effects and city-by-month fixed effects and are weighted by population, and standard errors are clustered at the city level. $N=30,134$ (a, low), 31,075 (a, high), 24,246 (b, low), 36,963 (b, high), 26,166 (c, low), and 35,043 (c, high). The separated observations are excluded (Methods).

and Peru ${ }^{43}$, and did not change in Greece ${ }^{31}$, Massachusetts (United States) ${ }^{25}$, Victoria (Australia) ${ }^{44}$, and China (outside Wuhan) $)^{45}$ (although suicide deaths in Nepal increased ${ }^{29}$ ), when these countries were placed under strict lockdowns. Furthermore, previous studies have frequently reported a drop followed by a delayed increase in suicide rates after national disasters, including after Hurricane Katrina in 2005 or the $9 / 11$ terrorist attack in 2001. The initial decline is called the pulling-together effect or honeymoon effect ${ }^{46-48}$ (Supplementary Note 4). Thus, the decline in suicides in the initial phase of this public health crisis is not surprising.

In Japanese contexts, several additional mechanisms could explain this decline during the initial outbreak. In response to the crisis, the national government provided several subsidies and benefits to citizens and enterprises (Supplementary Notes 1 and 2), which may have reduced economic distress. About $80 \%$ of the cash benefits were distributed to citizens before June (all citizens were eligible to receive cash benefits of $¥ 100,000$ (about US\$940), and suicide among individuals (employed, unemployed, and retired) decreased only before June (Fig. $4 \mathrm{a}-\mathrm{c}$ ). Additionally, claims for business subsidies grew rapidly between May and October, and the number of bankruptcies remained lower than the pre-pandemic level (Fig. 6e). The suicide rate among the self-employed did not increase during these periods.

Working hours for both full-time and part-time workers declined substantially (10\%-20\%) from April to May (Fig. 6f). Overwork and commuting are well-recognised risk factors for suicide, particularly among the working population in Japan ${ }^{49}$; hence, reduced working hours and work-from-home policies could have improved people's productivity, life satisfaction, and mental health ${ }^{50-52}$. This explanation is consistent with our finding of the largest decline in suicide rates among adults during the SOE (male adults, IRR $=0.79,95 \% \mathrm{CI}$ 0.73 to 0.85 ; female adults, $\mathrm{IRR}=0.73,95 \% \mathrm{CI} 0.65$ to 0.83 ), which comprise mainly the working population.

Finally, school closure may have reduced psychological burdens on children and adolescents, which could have resulted in suicide prevention. The suicide rate declined substantially among students during the nationwide school closure between March and April 2020 (IRR $=0.51,95 \%$ CI 0.34 to 0.77 ).

Our results offer a number of important insights on suicide mortality during the pandemic that may be relevant even after normal life resumes. First, the suicide trend in Japan could remain elevated in the long term. In the absence of effective pharmaceutical interventions, or with a delay in vaccine distribution, pandemic-related suicidal risk factors (for example, disease recurrence, social distancing, and economic downturns) would remain. While we have argued that massive government subsidies and benefits might have contributed to the prevention of suicides at the beginning of the epidemic, such generous financial support may not be sustainable in the long term. Thus, the overall suicide trends must be monitored, so that immediate policy responses can be considered.

In addition, our results suggest that to formulate effective prevention strategies amid the COVID-19 pandemic, customised approaches-rather than conventional ones-are needed. We found that, unlike normal economic circumstances, this pandemic disproportionately affects the psychological health of children, adolescents and females (especially housewives). Additionally, we found that only cities that previously had low suicide rates saw increases in suicide deaths during the pandemic. Therefore, the prevention strategy may need to target these vulnerable populations and locations.

Our results could also suggest routes towards suicide prevention even after normal life resumes. We argue that reduced working hours might have contributed to a reduction of suicide among adults during the SOE (April to May 2020), and school closure might have had a protective role for students. These effects highlight the need to identify the precise factors associated with workplaces and school sessions that could affect the psychological wellbeing of working populations and students.

Finally, we acknowledge the limitations of this study. First, while we have proposed potential mechanisms for the linkage between the pandemic and suicides, it is challenging to disentangle the contributions of each factor; this would require variation in the timing and intensity of each contributor (for example, disease prevalence, government interventions, economic shocks, financial support, and working conditions). However, the COVID-19 pandemic affects almost every community and citizen concurrently, and our analyses could not fully leverage such variation.

Moreover, we could not investigate the effects among specific subgroups of interest. For example, mental health consequences among healthcare professionals have been of great concern, as they are taking on extraordinary burdens during the pandemic ${ }^{53,54}$. Similarly, suicide effects among those with a high risk of fatality associated with COVID-19 (those with the presence of comorbidities) ${ }^{2,3}$, financial strain (low-skilled or low-income workers) ${ }^{36}$ or social isolation (including those who need mental healthcare or those with cognitive disorders) need to be carefully monitored.

Additionally, the suicide data may not be precise, as it is possible that some suicide deaths were not discovered or were reported with some delay. Moreover, suicide may have been misclassified as a 

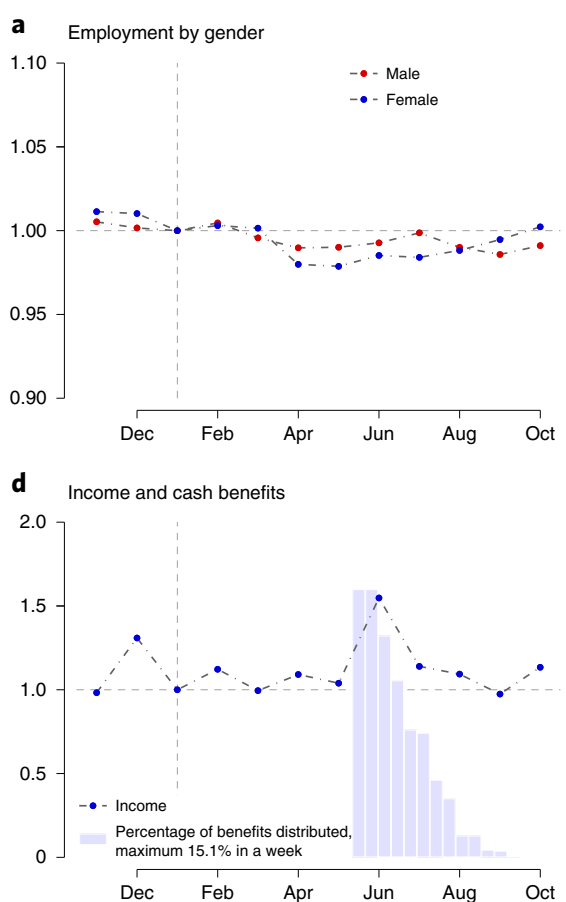
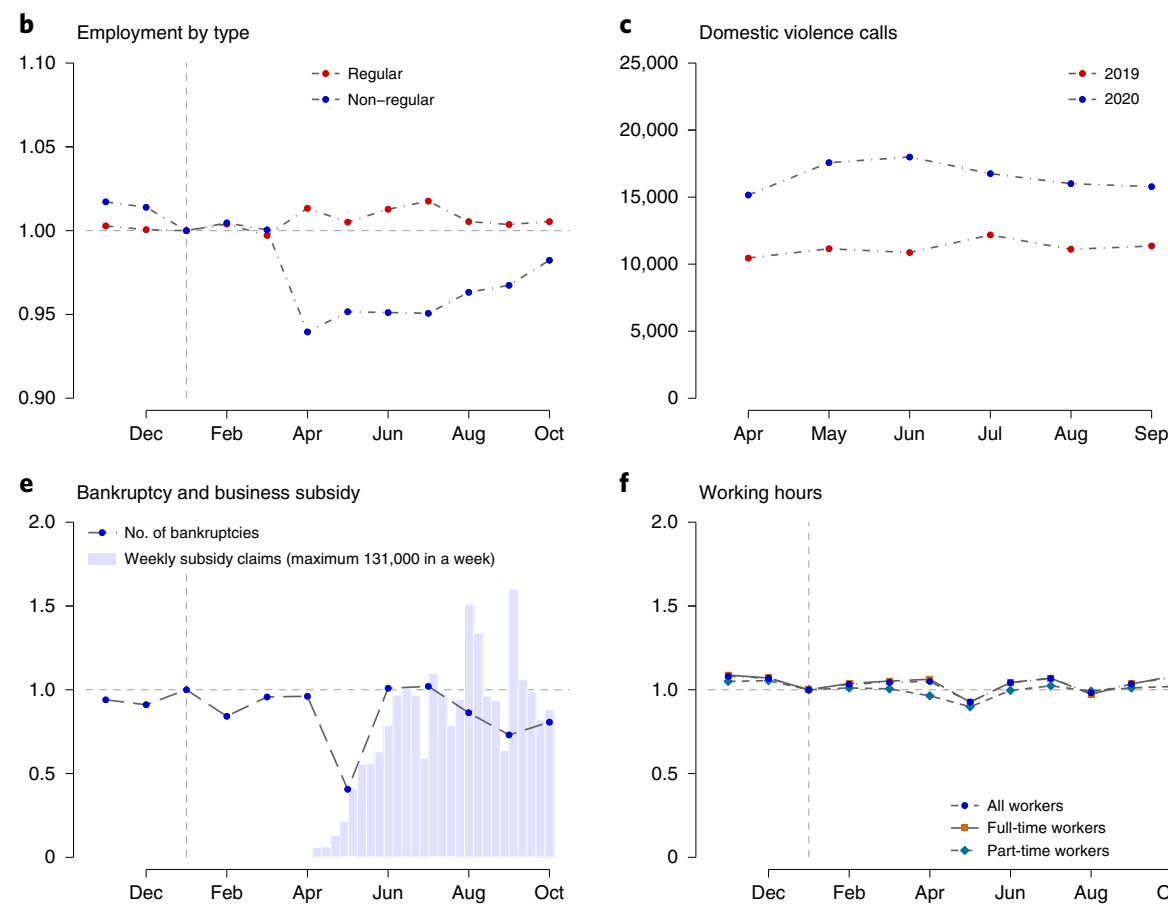

f

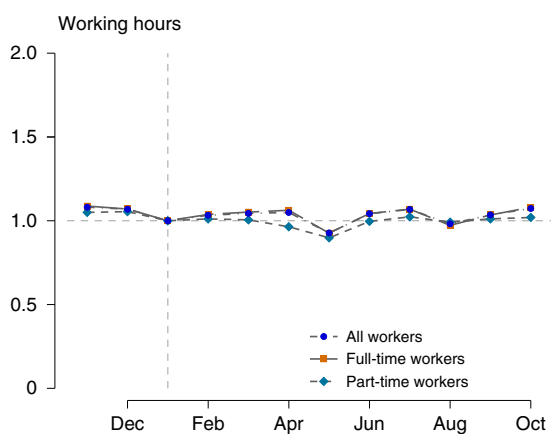

Fig. 6 | Mechanisms of the pandemic affecting suicide rate. $\mathbf{a}$, Change in employment rate for males and females. $\mathbf{b}$, Change in employment rate according to employment type (regular and non-regular workers). c, Number of calls for help related to domestic violence between April and December in 2019 and 2020. Data for October to March are not available. d, Mean household income excluding bonus payment. The bar graph shows the share of the cash benefits (total $¥ 100,000$ ) distributed in each week. e, Bankruptcies and claims for business subsidy. f, Mean working hours for full-time workers and part-time workers before and during the pandemic. In a,b,d-f, data are standardised to a value of 1.0 in January 2020.

different cause of death because the pandemic could have disrupted the reporting process.

Finally, we emphasise that the results of our study may not apply to other communities or countries because our study is founded on the unique Japanese public health, economic, cultural and social contexts. In particular, during our study period, the numbers of confirmed COVID-19 cases per population in Japan were only $2.9 \%$ of those in the United States and $12.7 \%$ of those in Germany (Supplementary Fig. 3a). Moreover, the Japanese government's health intervention was among the most lenient, based on 'request' rather than 'enforcement', ensuring a high degree of individual freedom (Supplementary Fig. 3b). Nevertheless, the national government provided generous fiscal support for households and companies, accounting for $10 \%$ of the annual GDP (Supplementary Fig. $3 c)$. These combined measures could make a difference in the extent of the epidemic's impact on mental wellbeing. Therefore, continuous assessment of the effect of the pandemic on each society is crucial to protect global psychological health and prevent increased suicide rates.

\section{Methods}

Data. We use city-by-month data on suicide records from November 2016 to October 2020, covering all suicide deaths among a total of 126 million citizens in Japan. The data are derived from suicide statistics published by the Ministry of Health, Labour, and Welfare, and they include information such as the number of suicides by age, gender, employment status, site, and day of the week ${ }^{55,56}$. The dataset includes 76,626 (monthly average 1,596) suicides in 1,848 cities $(N=88,512)$ with the average monthly suicide rate at 12.8 per million population. Males account for $68.2 \%$ of total suicides and male adults (between 20 and 69 years of age) contribute to about half of this total (50.2\%) (Supplementary Table 1). Using these data, we assess how suicide rates vary before and during the pandemic.

We also use other datasets to supplement our analysis, including the number of COVID-19 infections, weather conditions, and macroeconomic conditions (that is, bankruptcy, unemployment rates, consumption index, and diffusion index). Details for these data are available in Supplementary Note 1.
DID model. A central empirical challenge to estimating the effect of COVID-19 on suicide rates is to disentangle the effect of the pandemic from the long-term suicide trend and its seasonality. On average, the suicide rate has declined by 6.4\% from 2017 to 2019 (Extended Data Fig. 2; if data are extended, it declined by about $25 \%$ from 2013 to 2019). In addition, before the pandemic, the average suicide rate in February was $5.1 \%$ higher than that in January. These data imply that study design based on the before-after comparison could be problematic; if it compares the suicide levels before and during the COVID-19 outbreak ${ }^{29}$, the estimates might capture the seasonal trend (particularly between January and February); alternatively, if it compares the suicide level relative to past years in the same season ${ }^{26-28,30}$, the estimate might be confounded by a long-term ascending or descending trend.

The suicide trend and its seasonality also have to be accounted for at a disaggregated level, because they vary widely across locations (Extended Data Fig. 2). For instance, most regions had a declining suicide trend (Extended Data Fig. $2 \mathrm{bl}, \mathrm{cl}$ ), although it increased in some regions (Extended Data Fig. 2d1). Relatedly, we observe that the suicide rate in summer is higher in some locations (Extended Data Fig. 2b2), whereas this pattern is reversed in others (Extended Data Fig. 2e2). If we eliminate such location-specific trends and seasonality (we regress suicide rate on city-by-month and city-by-year fixed effects, and eliminate those effects), suicide patterns seem to be different from the observed trends (Extended Data Fig. 2a3-e3). These make the point that the time-series analysis, which compares national suicide trends, could easily generate biased estimates ${ }^{25-31}$. Instead, accurate estimation requires a quasi-experimental research design and harmonised data, by defining reasonable location-specific control conditions (counterfactual without the pandemic).

By leveraging our disaggregated but comprehensive dataset, we adopt the DID estimation with high-dimensional fixed effects. Our model is designed to overcome the empirical challenges. First, the model compared the difference in suicide rates before (November 2019 to January 2020) and during the virus outbreaks (February to October 2020) with the difference in the corresponding period in the previous three years (November 2016 to October 2019). Because the model focuses on the relative difference before and during the sudden pandemic within a year, the overall suicide level across years (the long-term suicide trend) is cancelled out. Second, we include city-by-month fixed effect and city-by-year fixed effect. These rich sets of fixed effects allow us to isolate the pandemic effects from the location-specific suicide trend and seasonality.

In particular, we specify the following model:

$$
Y_{i y m}=\alpha \text { Treat }_{\mathrm{y}} \times \text { Post }_{\mathrm{m}}+\mu_{\mathrm{im}}+\gamma_{\mathrm{iy}}+\varepsilon_{\text {iym }}
$$


where $Y$ denotes suicide rates in city $i$ in month $m$ in year $y$ (a year includes 12 months from November to October), and $\alpha$ is the parameter of interest, which denotes the impacts of the COVID-19 pandemic on suicide rates. Post ${ }_{m}$ is a binary variable that takes the value 1 if periods of observations corresponded to months between February (when the COVID-19 outbreak became salient and the national government launched the nationwide anti-contagion policies) and October. It takes a value of 0 if periods correspond to months from November to January, regarded as

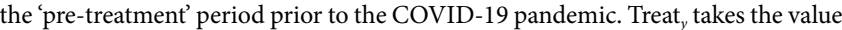
1 if the year is 2020 (November 2019 to October 2020) and 0 otherwise (November 2016 to October 2019). In this model, suicide trends between February and October from 2016 to 2019 serve as a control condition (counterfactual), after accounting for the level across years, with the assumption that we encounter only common shocks between the control and the treatment periods (during the pandemic)

We include city-by-month fixed effect and city-by-year fixed effect, denoted by $\mu_{i m}$ and $\gamma_{i y}$, respectively. City-by-month fixed effect flexibly controls for month-specific shocks in each city, such as seasonality in the suicide rate, monthly local events or climatic conditions ${ }^{57,58}$. City-by-year fixed effect controls for year-specific shocks in each city, such as macroeconomic trends, industrial or population structural changes, or suicide trends.

Suicide trends during the pandemic could vary across periods depending on the size of the ongoing outbreak, people's responses and the government's health interventions. Specifically, Japan faced two large COVID-19 outbreaks (Supplementary Note 2), and we might expect the suicide trend to vary in each wave. Unlike the time-series interrupted analysis, the DID desings enables us to estimate the time-varying effects flexibly (see Supplementary Note 5 and Supplementary Fig. 4 for details). Therefore, we estimate:

$$
Y_{\text {iym }}=\alpha_{f} \times \text { Treat }_{\mathrm{y}} \times \text { First }_{\mathrm{m}}+\alpha_{\mathrm{s}} \times \text { Treat }_{\mathrm{y}} \times \text { Second }_{\mathrm{m}}+\mu_{\text {im }}+\gamma_{\text {iy }}+\varepsilon_{\text {iym }}
$$

where First ${ }_{m}$ denotes the dummy variable, which takes the value 1 during months corresponding to the first outbreak (February to June) and Second ${ }_{m}$ takes the value 1 during months corresponding to the second outbreak (July to October). Similarly, we estimate how the suicide trends differ during the SOE (April and May 2020) and school closure (March and April 2020).

Our outcome variable of interest, suicide rate, is left-skewed and non-negative. Specifically, $58.7 \%$ of the city-by-month suicide rate takes a value of zero during our study period. Therefore, we use a Poisson-pseudo-maximum-likelihood estimator to specify equations (1) and (2) (refs. ${ }^{59,60}$ ). The adjusted model for Eq. (2) can be written as:

$$
Y_{\text {iym }}=\exp \left[\alpha_{f} \times \text { Treat }_{\mathrm{y}} \times \text { First }_{\mathrm{m}}+\alpha_{\mathrm{s}} \times \text { Treat }_{\mathrm{y}} \times \text { Second }_{\mathrm{m}}+\mu_{\text {im }}+\gamma_{\text {iy }}\right] \times \varepsilon_{\text {iym }} .
$$

We use the package ppmlhdfe to estimate the regression, with options 'weight', 'absorb' and 'cluster' in STATA v.16 to implement all the Poisson regression analyses ${ }^{59}$. We report the estimated coefficient in the form of IRRs. For this estimation, the necessary condition for the existence of the estimates is non-existence of the 'separated' observations ${ }^{60}$. Therefore, such observations are excluded from the analysis. Note that because more than $95 \%$ of the city-by-month suicide rates among children and adolescents are zero, we aggregated the data to the prefectural level for this specific cohort.

We cluster standard errors at the city level to allow arbitrary correlation over time within the same city. Additionally, all the regressions are weighted by population in 2018 so that cities with larger populations are given greater weights. Intuitively, these weights help to estimate the impact of the event on an average person instead of on an average city.

Event-study approach. The assumption for the DID estimator to be valid is that the pandemic period (February to October) in 2020 and the same periods in 20162019 would have parallel trends in suicide rates in the absence of the pandemic. If this assumption were not satisfied, the estimated parameter would be biased because the results could be driven by systematic differences between the treatment and control groups rather than the event of interest. To assess whether the parallel trends assumption would be reasonable, we adopt the event-study approach by fitting the following equation ${ }^{37,61}$ :

$$
Y_{i y m}=\exp \left[\sum_{k=-3, k \neq-1}^{8} \alpha_{k}\left(\text { Treat }_{y} \times \operatorname{Month}_{m, k}\right)+\mu_{i m}+\gamma_{i y}\right] \times \varepsilon_{i y m}
$$

where Month ${ }_{m}$ is 1 if the month corresponds to $k$, where $k=-1$ is set to be a month before the pandemic period (January). Intuitively, this casts the difference in suicide rates between 2020 season and 2016-2019 season in each month relative to $k=-1$; we expect the treatment group and control group to have a similar suicide rate before the disease outbreak becomes salient $(k<0)$ and we expect them to diverge after the outbreak $(k \geq 0)$.

Heterogeneity. We estimate the heterogeneity effects across different gender, age, job status, and geography. For age and gender, we re-estimate equation (3) by using suicide rate across gender and age groups (children and adolescents aged less than 20 years, the working-age population aged 20-69years, and older adults aged
70 year or more). For job status, we use suicide rate among the employed, retired, unemployed, self-employed, housewives, and students.

For heterogeneous analysis across geography, we re-estimate equation (3) using the subsamples. We use city-level base suicide rate (measured from November 2016 to January 2020), base income per capita (measured in 2018), and base share of urban population (measured in 2018) to classify the samples, in that, if the variable in a city is above its median, the corresponding city is classified as a high-suicide-rate, a high-income, or an urban city. Similarly, we use the prefectural level COVID-19 prevalence (measured by total confirmed cases per million population in October 2020), mobility restrictions (measured by change in Google Community Mobility at workplaces from January 2020 to after February 2020) and economic shocks (measured by the changes in unemployment between OctoberDecember 2019 and April-September 2020). We use prefectural level data for the sample classifications because the city-level data are not available. The details of these data are described in Supplementary Note 1.

Placebo test. We perform a placebo test ${ }^{37,62}$ to investigate whether impacts of the pandemic on suicide rates are driven by either common time trends or common shocks across different periods, using the following procedure. Using the data from November 2016 to October 2019, we randomly allocate treatment status to a year in the same period (February to June for the first outbreak, and July to October for the second outbreak) in each city and estimate the treatment effects analogously to equations (3) and (4). These equations can be written as:

$$
Y_{\text {iym }}=\exp \left[\alpha_{f}^{p} \times \text { Pl_Treat }_{\mathrm{y}} \times \text { First }_{\mathrm{m}}+\alpha_{\mathrm{s}}^{\mathrm{p}} \times \text { Pl_Treat }_{\mathrm{y}} \times \text { Second }_{\mathrm{m}}+\mu_{\text {im }}+\gamma_{\text {iy }}\right] \times \varepsilon_{\text {iym }}
$$

$$
Y_{\text {iym }}=\exp \left[\sum_{k=-3, k \neq-1}^{8} \alpha_{k}^{p}\left(\mathrm{Pl} \_ \text {Treat }_{y} \times \text { Month }_{m, k}\right)+\mu_{\text {im }}+\gamma_{\text {iy }}\right] \times \varepsilon_{\text {iym }}
$$

where Pl_Treat ${ }_{y}$ is 1 if the treatment status is allocated in both the equations. Then, we compare the placebo results to the real estimates. We repeat these procedures 1,000 times. If there is an event causing higher suicide incidence in a specific region in a pre-pandemic period (for example, cities in Tokyo prefecture in 2019 have unusually high suicide rates), our placebo results would include the spike in the estimated parameters. These results might imply that our main estimate is not driven by the disease outbreak, but by a random shock (or time trend) in some cities. We expect the placebo results (denoted by $\alpha_{f}^{p}, \alpha_{s}^{p}$ and $\alpha_{k}^{p}$ ) to not be statistically different from zero.

Back-of-the-envelope calculation. To estimate the increased or decreased deaths from suicide during the pandemic, we estimate the following equation:

$$
\begin{aligned}
\text { Suicide change }_{i, \text { treat,post }}= & \left(\widehat{\alpha_{f}} \times \text { Treat }_{\mathrm{y}} \times \text { First }_{\mathrm{m}}+\widehat{\alpha_{\mathrm{s}}} \times \text { Treat }_{\mathrm{y}} \times \text { Second }_{\mathrm{m}}\right) \\
& \times \text { Base suicide }_{\mathrm{i} \text {,treat,pre }}
\end{aligned}
$$

where Suicide change ${ }_{i \text {,treat,post }}$ denotes the predicted change in number of suicides in city $i$ in the treatment year (2020) during the pandemic period (after February). This is computed by estimated coefficients $\left(\widehat{\alpha_{f}}\right.$ and $\widehat{\alpha_{s}}$ ) derived by specifying equation (3) for the period during the first outbreak (Treat ${ }_{y} \times$ First $_{m}, 5$ months) and second outbreak (Treat ${ }_{y} \times$ Second $_{m}, 4$ months), and number of base suicide deaths

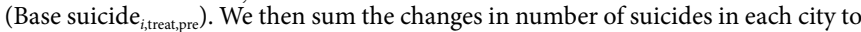
compute change on the national scale.

Reporting Summary. Further information on research design is available in the Nature Research Reporting Summary linked to this article.

\section{Data availability}

Data used in this paper are available at https://github.com/sokamoto-github/ Suicide-during-the-Covid-19-pandemic-in-Japan.

\section{Code availability}

Code used in this paper is available at https://github.com/sokamoto-github/ Suicide-during-the-Covid-19-pandemic-in-Japan.

Received: 8 September 2020; Accepted: 17 December 2020; Published online: 15 January 2021

\section{References}

1. Dong, E., Du, H. \& Gardner, L. An interactive web-based dashboard to track COVID-19 in real time. Lancet Infect. Dis. 20, 533-534 (2020).

2. Wu, J. T. et al. Estimating clinical severity of COVID-19 from the transmission dynamics in Wuhan, China. Nat. Med. 26, 506-510 (2020).

3. Williamson, E. J. et al. Factors associated with COVID-19-related death using OpenSAFELY. Nature 584, 430-436 (2020).

4. Hsiang, S. et al. The effect of large-scale anti-contagion policies on the COVID-19 pandemic. Nature 584, 262-267 (2020). 
5. Flaxman, S. et al. Estimating the effects of non-pharmaceutical interventions on COVID-19 in Europe. Nature 584, 257-261 (2020).

6. Wang, Y. et al. Remdesivir in adults with severe COVID-19: a randomised, double-blind, placebo-controlled, multicentre trial. Lancet 395, 1569-1578 (2020).

7. Wang, C. et al. A longitudinal study on the mental health of general population during the COVID-19 epidemic in China. Brain Behav. Immun. 87, 40-48 (2020)

8. Pierce, M. et al. Mental health before and during the COVID-19 pandemic: a longitudinal probability sample survey of the UK population. Lancet Psychiatry 7, P883-P892 (2020).

9. Gunnell, D. et al. Suicide risk and prevention during the COVID-19 pandemic. Lancet Psychiatry 7, 468-471 (2020)

10. Reger, M. A., Stanley, I. H. \& Joiner, T. E. Suicide mortality and coronavirus disease 2019-a perfect storm? JAMA Psychiatry https://doi.org/10.1001/ jamapsychiatry.2020.1060 (2020).

11. Pfefferbaum, B. \& North, C. S. Mental health and the Covid-19 pandemic. $N$. Engl. J. Med. 383, 510-512 (2020).

12. Christian, C., Hensel, L. \& Roth, C. Income shocks and suicides: causal evidence from Indonesia. Rev. Econ. Stat. 101, 905-920 (2019).

13. Oyesanya, M., Lopez-Morinigo, J. \& Dutta, R. Systematic review of suicide in economic recession. World J. Psychiatry 5, 243-254 (2015).

14. Kawohl, W. \& Nordt, C. COVID-19, unemployment, and suicide. Lancet Psychiatry 7, 389-390 (2020).

15. Wasserman, I. M. The impact of epidemic, war, prohibition and media on suicide: United States, 1910-1920. Suicide Life Threat. Behav. 22, 240-254 (1992).

16. Yip, P. S. F., Cheung, Y. T., Chau, P. H. \& Law, Y. W. The impact of epidemic outbreak. Crisis 31, 86-92 (2010).

17. Pierce, M. et al. Says who? The significance of sampling in mental health surveys during COVID-19. Lancet Psychiatry 7, 567-568 (2020).

18. Holmes, E. A. et al. Multidisciplinary research priorities for the COVID-19 pandemic: a call for action for mental health science. Lancet Psychiatry 7, 547-560 (2020).

19. Ammerman, B. A., Burke, T. A., Jacobucci, R. \& McClure, K. Preliminary investigation of the association between COVID-19 and suicidal thoughts and behaviors in the U.S. J. Psychiatr. Res. 134, 32-38 (2021).

20. Fitzpatrick, K. M., Harris, C. \& Drawve, G. How bad is it? Suicidality in the middle of the COVID-19 pandemic. Suicide Life Threat. Behav. 50, 1241-1249 (2020).

21. Halford, E. A., Lake, A. M. \& Gould, M. S. Google searches for suicide and suicide risk factors in the early stages of the COVID-19 pandemic. PLoS ONE 15, e0236777 (2020).

22. Iob, E., Steptoe, A. \& Fancourt, D. Abuse, self-harm and suicidal ideation in the UK during the COVID-19 pandemic. Br. J. Psychiatry 217, 1-4 (2020).

23. Li, D.-J. et al. COVID-19-related factors associated with sleep disturbance and suicidal thoughts among the Taiwanese public: a Facebook survey. Int. J. Environ. Res. Public Health 17, 4479 (2020).

24. Sinyor, M., Spittal, M. J. \& Niederkrotenthaler, T. Changes in suicide and resilience-related Google searches during the early stages of the COVID-19 pandemic. Can. J. Psychiatry 65, 741-743 (2020).

25. Faust, J. S. et al. Suicide deaths during the stay-at-home advisory in Massachusetts. Preprint at medRxiv https://doi. org/10.1101/2020.10.20.20215343 (2020)

26. Isumi, A. et al. Do suicide rates in children and adolescents change during school closure in Japan? The acute effect of the first wave of COVID-19 pandemic on child and adolescent mental health. Child Abus. Negl. 110, 104680 (2020)

27. Radeloff, D., et al. Trends in suicide rates during the COVID-19 pandemic restrictions in a major German city. Preprint at medRxiv https://doi. org/10.1101/2020.10.21.20187419 (2020).

28. Ueda, M., Nordström, R. \& Matsubayashi, T. Suicide and mental health during the COVID-19 pandemic in Japan. Preprint at medRxiv https://doi. org/10.1101/2020.10.06.20207530 (2020).

29. Pokhrel, S., Sedhai, Y. R. \& Atreya, A. An increase in suicides amidst the coronavirus disease 2019 pandemic in Nepal. Med. Sci. Law https://doi. org/10.1177/0025802420966501 (2020).

30. Qin, P. \& Mehlum, L. National observation of death by suicide in the first 3 months under COVID-19 pandemic. Acta Psychiatr. Scand. https://doi. org/10.1111/acps.13246 (2020).

31. Vandoros, S., et al. No evidence of increase in suicide in Greece during the first wave of Covid-19. Preprint at medRxiv https://doi. org/10.1101/2020.11.13.20231571 (2020).

32. IMF Fiscal Affairs Department. Fiscal Monitor Database of Country Fiscal Measures in Response to the COVID-19 Pandemic (IMF, 2020); https://www. imf.org/en/Topics/imf-and-covid19/Fiscal-Policies-Database-inResponse-to-COVID-19

33. World Development Indicators: Suicide Mortality Rate (Per 100,000 Population) (World Bank, accessed 6 August 2020); https://databank.worldbank.org/ reports.aspx . source $=2 \&$ series $=$ SH.STA.SUIC.P5\&country $=$
34. Vital Statistics (Ministry of Health, Labor and Welfare, accessed 6 August 2020); https://www.mhlw.go.jp/toukei/list/81-1.html

35. Koo, J. \& Cox, W. M. An economic interpretation of suicide cycles in Japan. Contemp. Econ. Pol. 26, 162-174 (2008).

36. Adams-Prassl, A., Boneva, T., Golin, M. \& Rauh, C. Inequality in the impact of the coronavirus shock: evidence from real time surveys. J. Public Econ. 189, 104245 (2020).

37. Leslie, E. \& Wilson, R. Sheltering in place and domestic violence: evidence from calls for service during COVID-19. J. Public Econ. 189, 104241 (2020).

38. Chang, S. S., Stuckler, D., Yip, P. \& Gunnell, D. Impact of 2008 global economic crisis on suicide: time trend study in 54 countries. Brit. Med. J. 347, f5239-f5239 (2013).

39. Alon, T. M., Doepke, M., Olmstead-Rumsey, J. \& Tertilt, M. The Impact of COVID-19 on Gender Equality NBER Working Paper Series https://doi. org/10.3386/w26947 (National Bureau of Economic Research, 2020).

40. Jacob, B. A. \& Lefgren, L. Are idle hands the devil's workshop? Incapacitation, concentration, and juvenile crime. Am. Econ. Rev. 93, 1560-1577 (2003).

41. Matsubayashi, T., Ueda, M. \& Yoshikawa, K. School and seasonality in youth suicide: evidence from Japan. J. Epidemiol. Community Health 70, 1122-1127 (2016).

42. Quarterly Suicide Death Registrations in England: 2001 to 2019 Registrations and Quarter 1 (Jan to Mar) to Quarter 2 (Apr to June) 2020 Provisional Data (Office for National Statistics, accessed 11 November 2020); https://www.ons. gov.uk/peoplepopulationandcommunity/birthsdeathsandmarriages/deaths/ bulletins/quarterlysuicidedeathregistrationsinengland/2001to2019registrations andquarter1 jantomartoquarter2aprtojune2020provisionaldata

43. Calderon-Anyosa, R. J. C. \& Kaufman, J. S. Impact of COVID-19 lockdown policy on homicide, suicide, and motor vehicle deaths in Peru. Prev. Med. 143, 106331 (2021).

44. Coroners Court Monthly Suicide Data Report: Report 2-5 October 2020 (Coroners Court of Victoria, 2020).

45. Qi, J. et al. Do lockdowns bring about additional mortality benefits or costs? Evidence based on death records from 300 million Chinese people. Preprint at medRxiv https://doi.org/10.1101/2020.08.28.20183699 (2020).

46. Bavel, J. J. V. et al. Using social and behavioural science to support COVID-19 pandemic response. Nat. Hum. Behav. 4, 460-471 (2020).

47. Benedek, D. M., Morganstein, J. C., Holloway, H. C. \& Ursano, R. J. in Kaplan and Sadock's Comprehensive Textbook of Psychiatry (eds Sadock, B. J. et al.) (Lippincott Williams \& Wilkins, 2017).

48. Kõlves, K., Kõlves, K. E. \& De Leo, D. Natural disasters and suicidal behaviours: a systematic literature review. J. Affect. Disord. 146, 1-14 (2013).

49. Inoue, K. \& Matsumoto, M. Karo jisatsu (suicide from overwork): a spreading occupational threat. Occup. Environ. Med. 57, 284a-285a (2000).

50. Bloom, N., Liang, J., Roberts, J. \& Ying, Z. J. Does working from home work? Evidence from a Chinese experiment. Q. J. Econ. 130, 165-218 (2015).

51. Mas, A. \& Pallais, A. Valuing alternative work arrangements. Am. Econ. Rev. 107, 3722-3759 (2017).

52. Kawaguchi, D., Naito, H. \& Yokoyama, I. Assessing the effects of reducing standard hours: regression discontinuity evidence from Japan. J. Jpn Int. Econ 43, 59-76 (2017).

53. Greenberg, N., Docherty, M., Gnanapragasam, S. \& Wessely, S. Managing mental health challenges faced by healthcare workers during covid-19 pandemic. BMJ 368, m1211 (2020).

54. Liu, Q. et al. The experiences of health-care providers during the COVID-19 crisis in China: a qualitative study. Lancet Glob. Health 8, e790-e798 (2020).

55. Suicide Statistics: Data on Suicide by Region (Ministry of Health, Labor and Welfare, accessed 10 July 2020); https://www.mhlw.go.jp/stf/seisakunitsuite/ bunya/0000140901.html

56. Summary of Suicide (National Police Agency, accessed 22 July 2020); https:// www.npa.go.jp/publications/statistics/safetylife/jisatsu.html

57. Carleton, T. A. Crop-damaging temperatures increase suicide rates in India. Proc. Natl Acad. Sci. USA 114, 8746-8751 (2017).

58. Burke, M. et al. Higher temperatures increase suicide rates in the United States and Mexico. Nat. Clim. Change 8, 723-729 (2018).

59. Correia, S., Guimarães, P. \& Zylkin, T. Fast Poisson estimation with high-dimensional fixed effects. Stata J. 20, 95-115 (2020).

60. Correia, S., Guimarães, P. \& Zylkin, T. Verifying the existence of maximum likelihood estimates for generalized linear models. Preprint at arXiv https:// arxiv.org/abs/1903.01633 (2019).

61. He, G., Pan, Y. \& Tanaka, T. The short-term impacts of COVID-19 lockdown on urban air pollution in China. Nat. Sustain. 3, 1005-1011 (2020).

62. Heft-Neal, S. et al. Dust pollution from the Sahara and African infant mortality. Nat. Sustain. 3, 863-871 (2020).

\section{Acknowledgements}

We thank Y. Pan and seminar participants at the 23rd Tokyo Labour Economics Workshop for their insightful comments. T.T. thanks the Bai Xian Asia Institute for the scholarship support as a Bai Xian Scholar. S.O. is supported by a postdoctoral fellowship of the Japan Society for the Promotion of Science (no. 20J00394) 
and the Murata Science Foundation. These funders were not engaged in the conceptualisation, design, data collection, analysis, decision to publish or preparation of the manuscript.

\section{Author contributions}

S.O. and T.T. conceptualised the study and carried out initial planning. T.T. retrieved and constructed the dataset. T.T. carried out the statistical analysis, which was refined by S.O. for the final version. S.O. prepared the first draft of the report, which was revised by T.T. Both authors reviewed and contributed to the final draft and approved the final version for publication.

\section{Competing interests}

The authors declare no competing interests.

\section{Additional information}

Extended data is available for this paper at https://doi.org/10.1038/s41562-020-01042-z.

Supplementary information The online version contains supplementary material available at https://doi.org/10.1038/s41562-020-01042-z.

Correspondence and requests for materials should be addressed to S.O.

Peer review information Nature Human Behaviour thanks Jane Pirkis and the other, anonymous, reviewer(s) for their contribution to the peer review of this work. Peer reviewer reports are available. Primary Handling Editor: Charlotte Payne.

Reprints and permissions information is available at www.nature.com/reprints.

Publisher's note Springer Nature remains neutral with regard to jurisdictional claims in published maps and institutional affiliations.

(c) The Author(s), under exclusive licence to Springer Nature Limited 2021 
a. Total

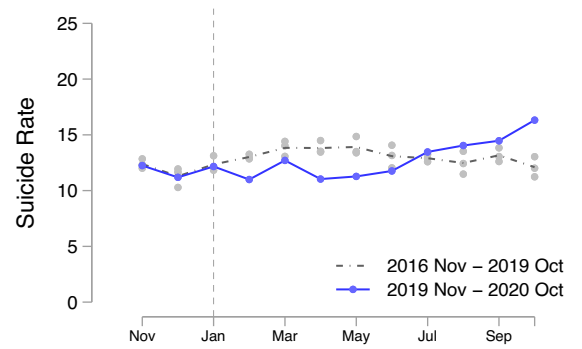

d. Age below 20

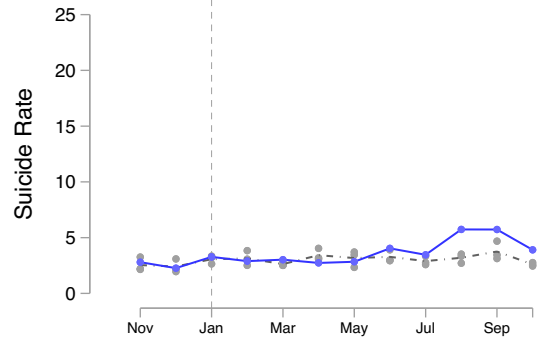

b. Male

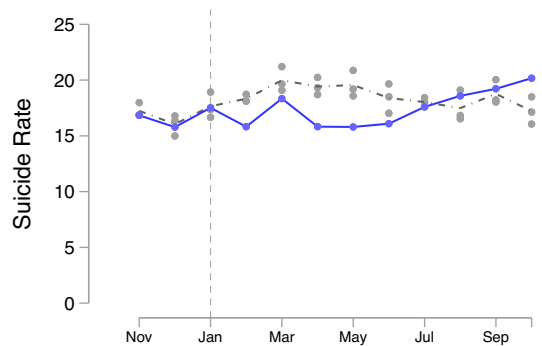

e. Age $20 \sim 69$

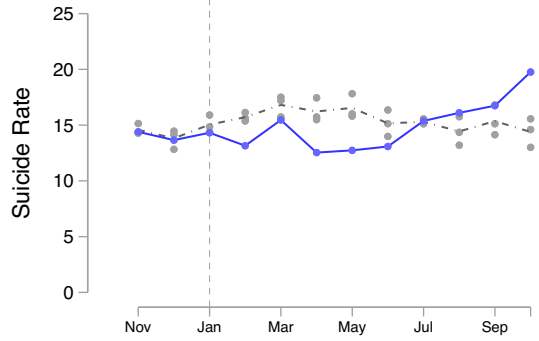

c. Female

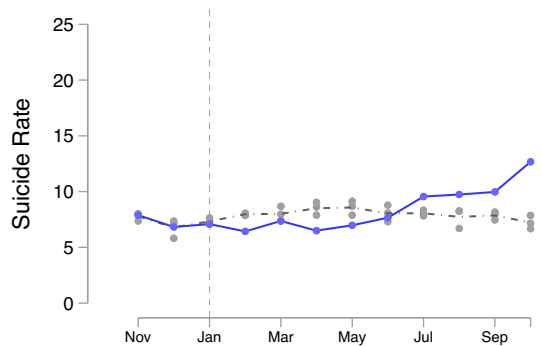

f. Age over 70

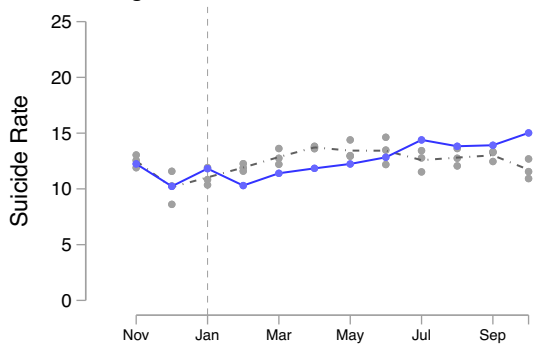

Extended Data Fig. 1 | The trend of suicide across gender and age groups. Panels a-f show the trend of per-month suicide rates per million from November 2016 to October 2020. The grey line represents the mean suicides rates for three years before the pandemic year (November 2016 to October 2019) with a grey circle denoting suicide rates each year. The light blue line shows the suicides rates from November 2019 to October 2020. 
a1. Suicide trend: all

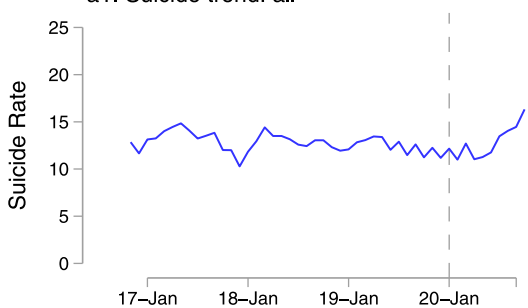

b1. Suicide trend: Hokkaido

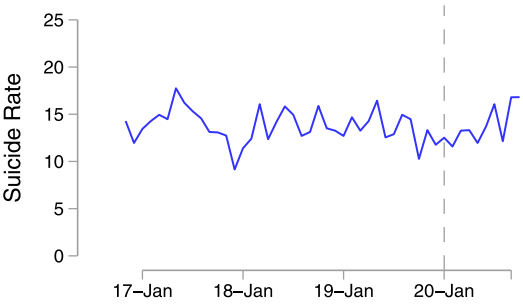

c1. Suicide trend: Tokyo

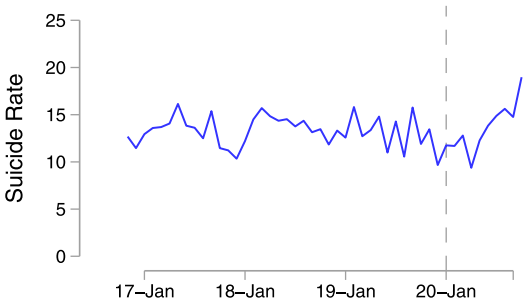

d1. Suicide trend: Nara

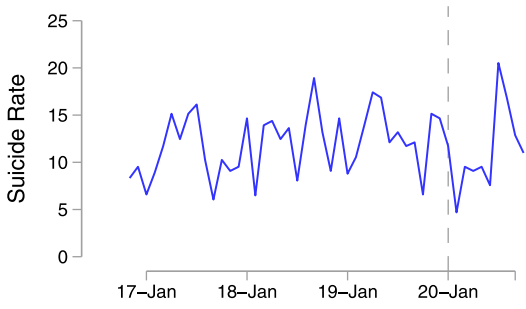

e1. Suicide trend: Fukuoka

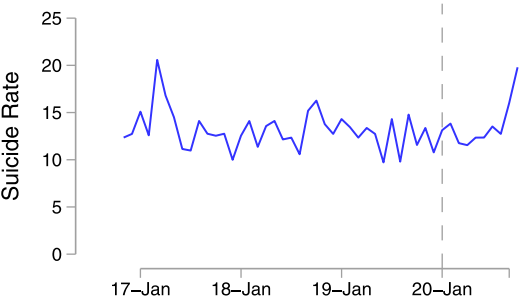

a2. Seasonality: all

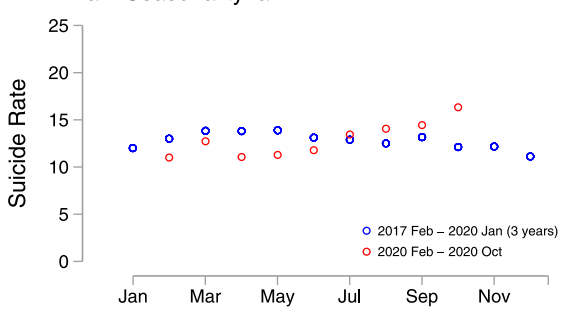

b2. Seasonality: Hokkaido

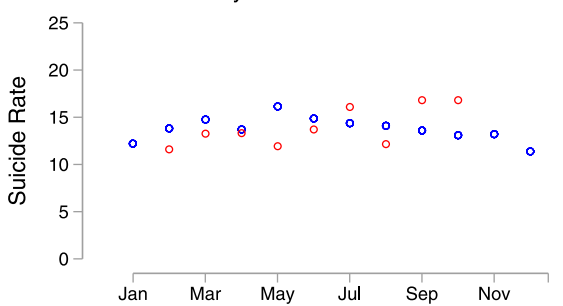

c2. Seasonality: Tokyo

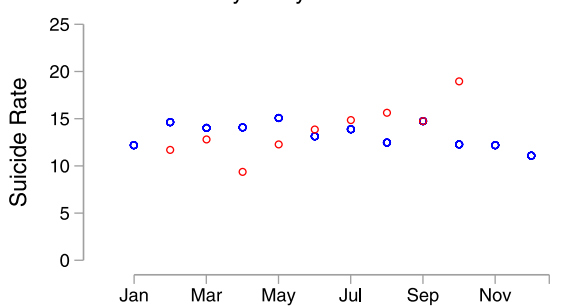

d2. Seasonality: Nara

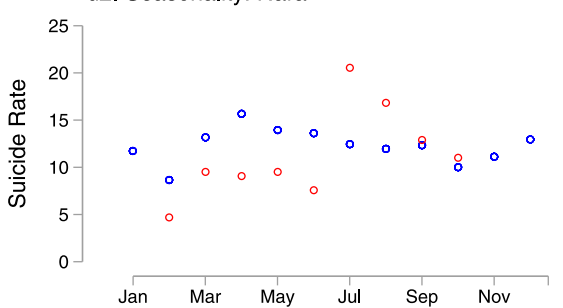

e2. Seasonality: Fukuoka

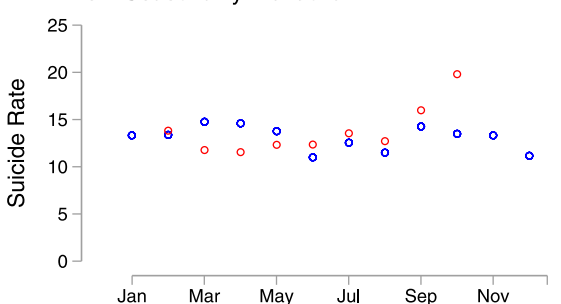

a3. Remove trend \& seasonality: all

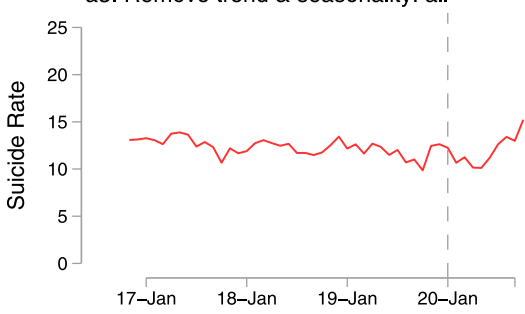

b3. Remove trend \& seasonality: Hokkaido

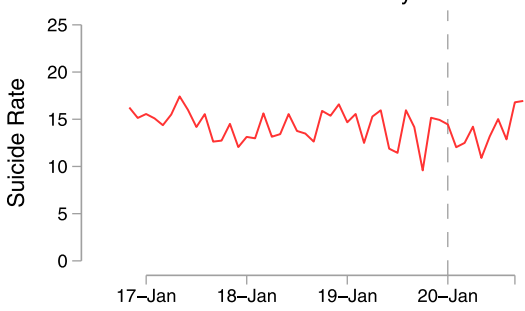

c3. Remove trend \& seasonality: Tokyo

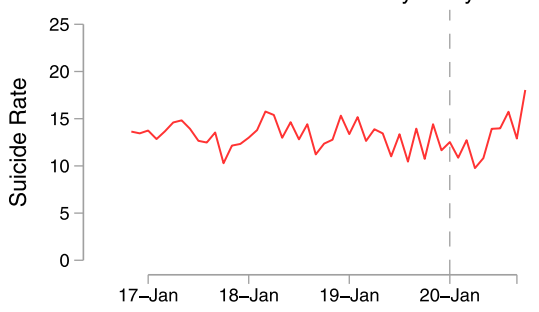

d3. Remove trend \& seasonality: Nara

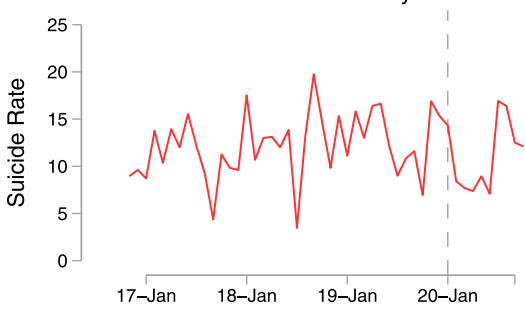

e3. Remove trend \& seasonality: Fukuoka

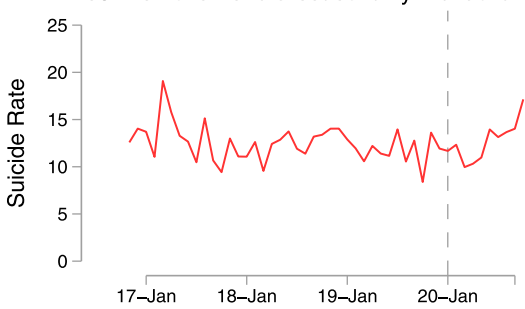

Extended Data Fig. 2 | The trend and seasonality of suicide across regions. Panels a1-e1 show the observed per-month suicide rate per million in each prefecture. Panels a2-e2 represent the seasonal suicide trend before the pandemic (Feb 2017- Jan 2020, three years) and during the pandemic. Panels a3-e3 describe the suicide trends after eliminating the effects of suicide trend and seasonality. To do so, we regress suicide rate on city-by-month fixed effects, and city-specific suicide linear time trends, and eliminate these effects. Panel a uses all observations (1,848 cities), Panel $\mathbf{b}$ uses the cities in Hokkaido prefecture (177 cities), Panel c uses Tokyo prefecture (57 cities), Panel d uses Nara prefecture (39 cities), and Panel e uses Fukuoka prefecture (73 cities). 


\section{a. Placebo DID estimates}

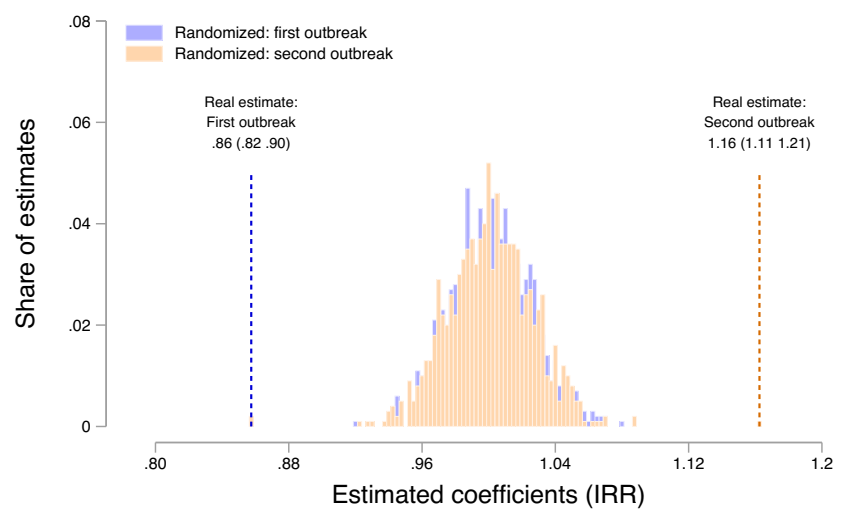

b. Placebo event study estimates

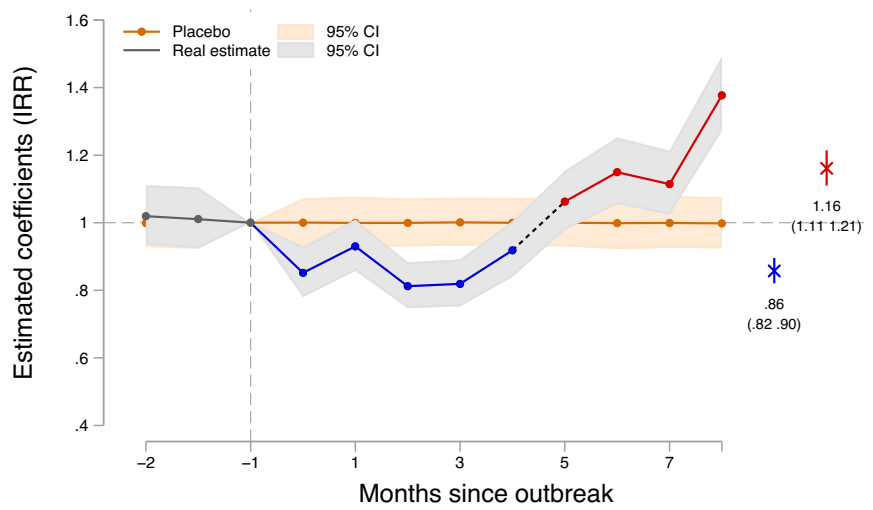

Extended Data Fig. 3 | Placebo test. Panel a represents the results of the placebo test. Using samples from 2016 to 2019 , we first randomly allocate treatment dummies within the city across the years. We then regress the suicide rate on the placebo treatment 1,000 times. The mean effect using the placebo sample is around zero, and the estimate of the first outbreak is much smaller than $95 \% \mathrm{Cl}$ of the placebo results and that of second outbreak is much larger than $95 \% \mathrm{Cl}$ of the placebo results. Panel $\mathbf{b}$ repeats event study regression using the placebo samples analogously. The estimated coefficient is close to zero in all periods. All regressions include city-by-year fixed effects and city-by-month fixed effects and are weighted by population, and standard errors are clustered at the city level. $N=61,209$ (b). Each regression for 1,000 times has different number of observations (a). The separated observations are dropped (see Methods). 
a. Predicted effects of COVID-19 on suicide rate

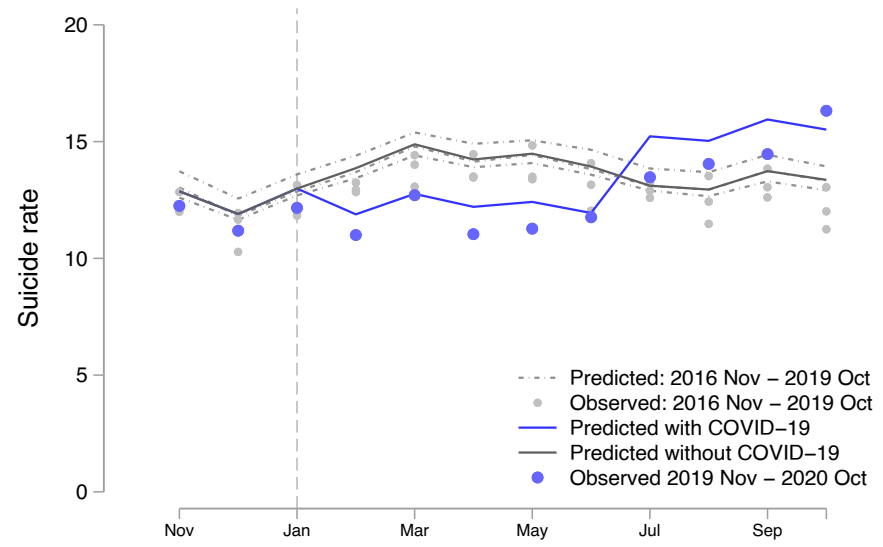

b. Estimated number of suicide change

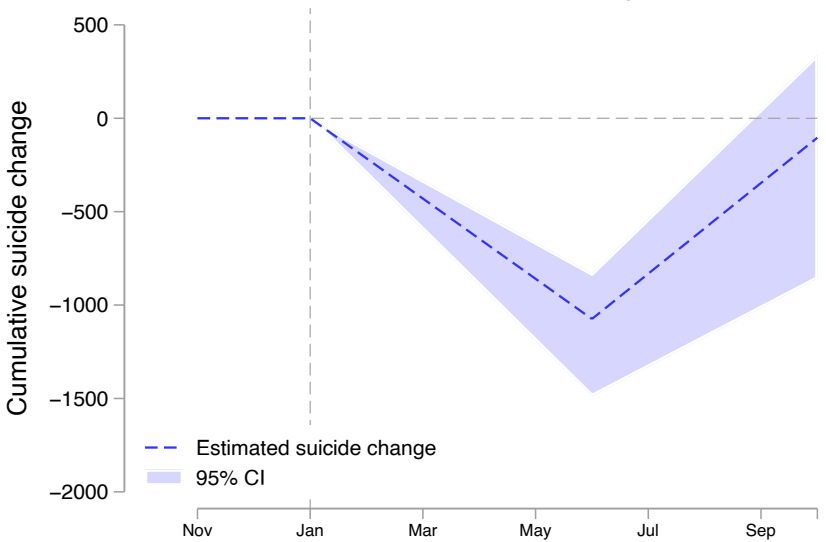

Extended Data Fig. 4 | Back-of-the-envelope calculation for the predicted deaths from suicide. Panel a describes the predicted change in suicide rate (per-month per million) with COVID-19 and otherwise. The difference between the grey line (without COVID-19) and the blue line (with COVID-19) represents the effects of the pandemic. Panel $\mathbf{b}$ demonstrates the predicted increased or decreased deaths by suicide across periods. The blue dashed line represents the points estimate, while the light blue area shows the $95 \% \mathrm{Cl}$. All regressions include city-by-year fixed effects and city-by-month fixed effects and are weighted by population, and standard errors are clustered at the city level. $N=61,209$ (a). The separated observations are dropped (see Methods). 
a. Intensity of health interventions

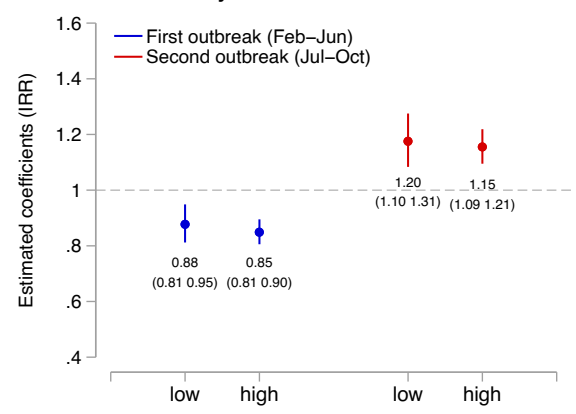

b. Economic shock

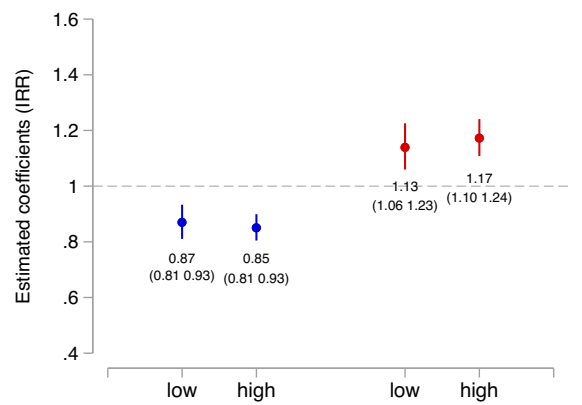

c. Urban population (\%)

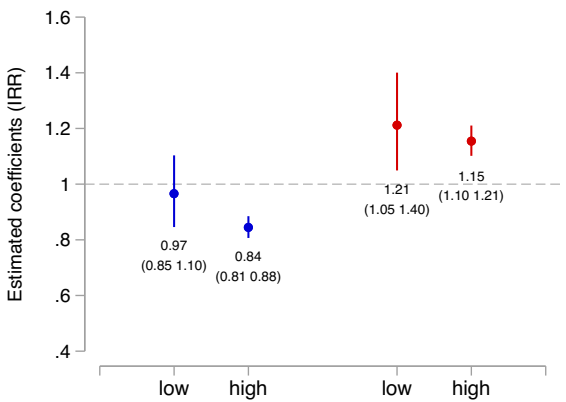

Extended Data Fig. 5 | Heterogeneous effects of the COVID-19 pandemic across geography. This graph describes the results of the effects of the pandemic on suicide during the first and second outbreaks across locations with different prefecture-level intensity of health interventions (panel a), prefecture-level economic shock (b), and base share of urban population (c). The intensity of health intervention is measured by the level of decline in the Google Community Mobility index at workplaces, and economic shock by the changes in unemployment rate. If the variable in a prefecture or city is lower than the median, the prefecture or city is defined as 'low'. All regressions include city-by-year fixed effects and city-by-month fixed effects and are weighted by population, and standard errors are clustered at the city level. $N=23,530$ (a, low), 37,679 (a, high), 26,004 (b, low), 35,205 (b, high), 18,784 (c, low), 42,425 (c, high). The separated observations are dropped (see Methods). 


\section{Reporting Summary}

Nature Research wishes to improve the reproducibility of the work that we publish. This form provides structure for consistency and transparency in reporting. For further information on Nature Research policies, see our Editorial Policies and the Editorial Policy Checklist.

\section{Statistics}

For all statistical analyses, confirm that the following items are present in the figure legend, table legend, main text, or Methods section.

n/a Confirmed

$\bigotimes$ The exact sample size $(n)$ for each experimental group/condition, given as a discrete number and unit of measurement

$\square$ A statement on whether measurements were taken from distinct samples or whether the same sample was measured repeatedly

$\square$ The statistical test(s) used AND whether they are one- or two-sided

$\square$ Only common tests should be described solely by name; describe more complex techniques in the Methods section.

$\square$ A description of all covariates tested

$\square$ \A description of any assumptions or corrections, such as tests of normality and adjustment for multiple comparisons

$\square$ A full description of the statistical parameters including central tendency (e.g. means) or other basic estimates (e.g. regression coefficient)

$\triangle$ AND variation (e.g. standard deviation) or associated estimates of uncertainty (e.g. confidence intervals)

$\varnothing$ For null hypothesis testing, the test statistic (e.g. $F, t, r$ ) with confidence intervals, effect sizes, degrees of freedom and $P$ value noted

Give P values as exact values whenever suitable.

Х $\square$ For Bayesian analysis, information on the choice of priors and Markov chain Monte Carlo settings

$\square$ \ For hierarchical and complex designs, identification of the appropriate level for tests and full reporting of outcomes

$\square \bigotimes$ Estimates of effect sizes (e.g. Cohen's $d$, Pearson's $r$ ), indicating how they were calculated

Our web collection on statistics for biologists contains articles on many of the points above.

\section{Software and code}

Policy information about availability of computer code

Data collection Computer code is not used to collect data

Data analysis Stata V16 is used.

For manuscripts utilizing custom algorithms or software that are central to the research but not yet described in published literature, software must be made available to editors and reviewers. We strongly encourage code deposition in a community repository (e.g. GitHub). See the Nature Research guidelines for submitting code \& software for further information.

\section{Data}

Policy information about availability of data

All manuscripts must include a data availability statement. This statement should provide the following information, where applicable:

- Accession codes, unique identifiers, or web links for publicly available datasets

- A list of figures that have associated raw data

- A description of any restrictions on data availability

Data used in this paper are available at https://github.com/sokamoto-github/Suicide-during-the-Covid-19-pandemic-in-Japan. 


\section{Field-specific reporting}

Please select the one below that is the best fit for your research. If you are not sure, read the appropriate sections before making your selection.
$\square$ Life sciences
Behavioural \& social sciences
Ecological, evolutionary \& environmental sciences

For a reference copy of the document with all sections, see nature.com/documents/nr-reporting-summary-flat.pdf

\section{Behavioural \& social sciences study design}

All studies must disclose on these points even when the disclosure is negative.

\begin{tabular}{|c|c|}
\hline Study description & This study assesses if suicide mortality changed during the COVID-19 pandemic in Japan \\
\hline Research sample & This research sample consists of all suicide death in Japan from July 2016 to October 2020. \\
\hline Sampling strategy & There is no sampling strategy because our data includes all suicide deaths in Japan \\
\hline Data collection & Data were collected from the publicly available information. See the data and supplementary note 1 for the data sources. \\
\hline Timing & Our data include suicide from July 2016 to October 2020. \\
\hline Data exclusions & No data were excluded \\
\hline Non-participation & No participation was dropped \\
\hline Randomization & This study did not conduct any experiment. Thus, no randomization was conducted. \\
\hline
\end{tabular}

\section{Reporting for specific materials, systems and methods}

We require information from authors about some types of materials, experimental systems and methods used in many studies. Here, indicate whether each material, system or method listed is relevant to your study. If you are not sure if a list item applies to your research, read the appropriate section before selecting a response.

\begin{tabular}{|c|c|}
\hline $\mathrm{n} / \mathrm{a}$ & Involved in the study \\
\hline Х & $\square$ Antibodies \\
\hline Х & $\square$ Eukaryotic cell lines \\
\hline$\bigotimes$ & $\square$ Palaeontology and archaeology \\
\hline$\bigotimes$ & $\square$ Animals and other organisms \\
\hline$\bigotimes$ & $\square$ Human research participants \\
\hline$\bigotimes$ & $\square$ Clinical data \\
\hline Х & $\square$ Dual use research of concern \\
\hline
\end{tabular}

Methods $\mathrm{n} / \mathrm{a}$ Involved in the study

\ $\square$ ChIP-seq

Х $\square$ Flow cytometry

$\bigotimes \mid \square$ MRI-based neuroimaging 